NASA Technical Memorandum 106818

AIAA-95-0763

\title{
The Space-Time Solution Element Method- A New Numerical Approach for the Navier-Stokes Equations
}

James R. Scott and Sin-Chung Chang

Lewis Research Center

Cleveland, Ohio

Prepared for the 33rd Aerospace Sciences Meeting sponsored by the American Institute of Aeronautics and Astronautics Reno, Nevada, January 9-12, 1995

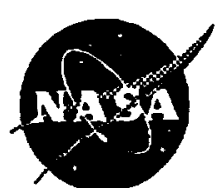



. 


\title{
THE SPACE-TIME SOLUTION ELEMENT METHOD - A NEW NUMERICAL APPROACH FOR THE NAVIER-STOKES EQUATIONS
}

\author{
James R. Scott* and Sin-Chung Changt \\ NASA Lewis Research Center \\ Cleveland, Ohio
}

\begin{abstract}
This paper is one of a series of papers describing the development of a new numerical method for the Navier-Stokes equations. Unlike conventional numerical methods, the current method concentrates on the discrete simulation of both the integral and differential forms of the Navier-Stokes equations. Conservation of mass, momentum, and energy in space-time is explicitly provided for through a rigorous enforcement of both the integral and differential forms of the governing conservation laws. Using local polynomial expansions to represent the discrete primitive variables on each cell, fluxes at cell interfaces are evaluated and balanced using exact functional expressions. No interpolation or flux limiters are required. Because of the generality of the current method, it applies equally to the steady and unsteady Navier-Stokes equations. In this paper, we generalize and extend the authors' 2-D, steady-state implicit scheme. A general closure methodology is presented so that all terms up through a given order in the local expansions may be retained. The scheme is also extended to nonorthogonal Cartesian grids. Numerous flow fields are computed and results are compared with known solutions. The high accuracy of the scheme is demonstrated through its ability to accurately resolve developing boundary layers on coarse grids. Finally, we discuss applications of the current method to the unsteady Navier-Stokes equations.
\end{abstract}

*Research Scientist, Internal Fluid Mechanics Division, Member AIAA

$\nmid$ Research Scientist, Internal Fluid Mechanics Division

Copyright (C)1995 by the American Institute of Aeronautics and Astronautics, Inc. No copyright is asserted in the United States under Title 17, U.S. Code. The U.S. Government has a royalty-free license to exercise all rights under the copyright claimed herein for government purposes. All other rights are reserved by the copyright owner.

\section{Introduction}

This paper is concerned with the continued development of a new numerical methodt for solving the Navier-Stokes equations. ${ }^{1-3}$ The distinguishing features of the current method have been previously described, and may be summarized as follows: The current method (i) provides for a unified treatment of space and time; (ii) represents the local discrete primitive variables through Taylor series expansions that identically satisfy both the integral and differential forms of the Navier-Stokes equations; (iii) balances fluxes across cell interfaces as an integral part of the numerical formulation; (iv) evaluates fluxes at cell boundaries using exact functional expressions (to the order of accuracy of the local expansions); and ( $v$ ) solves explicitly for the unknown derivatives of the local discrete primitive variables.

Scott and Chang ${ }^{1}$ developed a Newton's method implicit scheme for the two-dimensional, steady Navier-Stokes equations. Calculations of laminar flow in a channel showed that the developing boundary layer could be accurately resolved using as few as six cells per channel width. Scott ${ }^{3}$ extended the internal flow scheme to external flows, and showed that the boundary layer on a finite flat plate could be accurately resolved using only ten cells from the wall to the free stream. Chang ${ }^{2}$ developed an explicit scheme for the one-dimensional, unsteady Navier-Stokes equations, and presented numerical results for the shock tube problem with viscosity. It was shown that the shock and contact discontinuities could be crisply resolved without the use of flux limiters or weighting functions.

In this paper we are concerned with the further development of the steady-state flux conservation scheme first presented in Reference [1]. Here we

‡Known most completely as The Space-Time Conservation and Solution Element Method, and frequently abbreviated as the STS Method, Conservation Element Method, Solution Element Method, or the CESE Method. 
generalize the scheme by (i) providing a general closure methodology so that all discrete unknowns of the same order in the local expansions may always be retained and (ii) by extending the scheme to nonorthogonal Cartesian grids. Calculations of a variety of flow fields indicate that developing laminar boundary layers can be accurately resolved on grids which are much coarser than those employed by conventional numerical methods.

In the next section we begin by considering the general problem of steady-state flux conservation in two space dimensions. Following that, we summarize the steady-state flux conservation scheme as originally presented in [1] and [3]. Then we show how the scheme may be generalized and extended to general flow fields and nonorthogonal grids. Finally, we discuss applications of the present approach to the unsteady NavierStokes equations.

\section{Steady-State Flux Conservation in Tro Space Dimensions}

We begin by considering the system of integral conservation laws

$$
\oint_{s(V)} \overrightarrow{\mathbf{h}}_{\mathrm{m}} \cdot \overrightarrow{d s}=0
$$

in $E_{2}$, where $\overrightarrow{\mathrm{h}}_{\mathrm{m}}, \mathrm{m}=1, \ldots, \mathrm{M}$ is a steady-state flux density vector which is a function of $M$ intermediate (primitive) variables. $S(V)$ is an orientable surface inclosing an arbitrary volume $V$ in two-dimensional space, and $\overrightarrow{d s}$ is the outward area vector which is normal to $S(V)$. Equation (2.1) is the governing equation for steady-state flux conservation in two space dimensions.

Suppose that (2.1) holds for any $V$ in a fixed domain $\mathcal{D}$ in $E_{2}$, and that each $\overrightarrow{\mathrm{h}}_{\mathrm{m}}$ varies smoothly throughout $\mathcal{D}$. Then one obtains, by way of the divergence theorem, the differential conservation law

$$
\vec{\nabla} \cdot \vec{h}_{m}=0
$$

where $\vec{\nabla}=\left(\frac{\partial}{\partial x}, \frac{\partial}{\partial y}\right)$. If information about the behavior of the $\vec{h}_{m}$ (or their intermediate variables) on the boundary $B(\mathcal{D})$ of $\mathcal{D}$ is also known, then equation (2.2), along with the known boundary information, represents a steady-state boundary value problem. We will subsequently refer to equation (2.2) and its associated boundary conditions as boundary value problem (2.2).

Suppose that $\mathcal{D}$ is the rectangular region shown in Figure 1 , and that $\vec{h}_{\mathrm{m}}, \mathrm{m}=1, \ldots, \mathrm{M}$ is the exact solution to boundary value problem (2.2). In addition, let eaci $\vec{h}_{\mathrm{m}}$ be analytic throughout $\mathcal{D}$. (From here on, we drcp the $m$ subscript for clarity.) Then near any $\left(x_{0}, y_{0}\right)$ in $\mathcal{D}, \vec{h}_{\mathrm{m}} \stackrel{\text { def }}{=} \vec{h}=\left(h^{x}, h^{y}\right)$ may be expressed by the uniformly convergent Taylor series expansions ${ }^{3}$

$$
\begin{gathered}
h^{x}(x, y)=h^{x}\left(x_{0}, y_{0}\right)+\frac{\partial h^{x}}{\partial x}\left(x_{0}, y_{0}\right)\left(x-x_{0}\right) \\
+\frac{\partial h^{x}}{\partial y}\left(x_{0}, y_{0}\right)\left(y-y_{0}\right)+\ldots \\
=\sum_{n=0}^{\infty} \sum_{k=0}^{n}\left[\frac{\partial^{n} h^{x}}{\partial x^{n-k} \partial y^{k}}\left(x_{0}, y_{0}\right)\right] \frac{\left(x-x_{0}\right)^{n-k}}{(n-k) !} \frac{\left(y-y_{0}\right)^{k}}{k !} \\
h^{y}(x, y)=h^{y}\left(x_{0}, y_{0}\right)+\frac{\partial h^{y}}{\partial x}\left(x_{0}, y_{0}\right)\left(x-x_{0}\right) \\
\quad+\frac{\partial h^{y}}{\partial y}\left(x_{0}, y_{0}\right)\left(y-y_{0}\right)+\ldots \\
=\sum_{n=0}^{\infty} \sum_{k=0}^{n}\left[\frac{\partial^{n} h^{y}}{\partial x^{n-k} \partial y^{k}}\left(x_{0}, y_{0}\right)\right] \frac{\left(x-x_{0}\right)^{n-k}}{(n-k) !} \frac{\left(y-y_{0}\right)^{k}}{k !} .
\end{gathered}
$$

If the infinite series (2.3) - (2.4) is truncated at some point, then it becomes an local approximation to the exact solution $\vec{h}$.

Let $\mathcal{D}$ be discretized by the rectangular mesh shown in Figure 2, and let $\left(x_{i}, y_{j}\right)$ denote the center point of each cell. Let $\left(\vec{h}_{2}\right)_{i, j}$ denote the order-two Taylor series expansion of $\vec{h}$ about the point $\left(x_{i}, y_{j}\right)$. Then

$$
\begin{gathered}
\left(h_{2}^{x}\right)_{i, j}= \\
=\sum_{n=0}^{2} \sum_{k=0}^{n}\left[\frac{\partial^{n} h^{x}}{\partial x^{n-k} \partial y^{k}}\left(x_{i}, y_{j}\right)\right] \frac{\left(x-x_{i}\right)^{n-k}}{(n-k) !} \frac{\left(y-y_{j}\right)^{k}}{k !}
\end{gathered}
$$

and

$$
\left(h_{2}^{y}\right)_{i, j}=
$$

$$
=\sum_{n=0}^{2} \sum_{k=0}^{n}\left[\frac{\partial^{n} h^{y}}{\partial x^{n-k} \partial y^{k}}\left(x_{i}, y_{j}\right)\right] \frac{\left(x-x_{i}\right)^{n-k}}{(n-k) !} \frac{\left(y-y_{j}\right)^{k}}{k !} \text {. }
$$

As an approximation to $\vec{h}$ on the $(i, j)$ th cell, it can be shown that ${ }^{3}$ the error of $\left(\vec{h}_{2}\right)_{i, j}$ is bounded by $\frac{M_{i, i}}{4}[\max (\Delta x, \Delta y)]^{3}$, where $M_{i, j}$ is a local bound on the third derivatives of $\vec{h}$. Thus, $\left\{\left(\vec{h}_{2}\right)_{i, j}, i=\right.$ $\left.1, \ldots, N_{i}, j=1, \ldots, N_{j}\right\}$ is a discrete approximation to the exact solution $\vec{h}$ on $\mathcal{D}$.

The local Taylor series expansion $\left(\vec{h}_{2}\right)_{i, j}$ is a solution of the differential conservation law (2.2), since its divergence is identically zero. ${ }^{3}$ This follows from 
the fact that (2.3) - (2.4) also satisfies equation (2.2). Since $\left(\vec{h}_{2}\right)_{i, j}$ is a second-order polynomial that provides the exact values of $\vec{h}$ and its derivatives through order two at the point $\left(x_{i}, y_{j}\right)$, it will be referred to as the exact second-order solution of equation (2.2).

Since the derivatives of $\vec{h}$ are well-defined throughout $\mathcal{D}$, we may now consider the approximate second-order solution $\vec{h}_{2}$ to boundary value problem (2.2), where

$$
\overrightarrow{\underline{h}}_{2} \stackrel{\text { def }}{=} \overrightarrow{\underline{h}}=\left(\underline{h}^{x}, \underline{h}^{y}\right)
$$

with

$$
\begin{gathered}
\text { with } \\
\stackrel{h^{x}(x, y ; i, j)}{\stackrel{\text { def }}{=}} \\
h_{0,0}^{x}+h_{1,0}^{x}\left(x-x_{i}\right)+h_{0,1}^{x}\left(y-y_{j}\right)+ \\
h_{2,0}^{x}\left(x-x_{i}\right)^{2}+h_{1,1}^{x}\left(x-x_{i}\right)\left(y-y_{j}\right)+h_{0,2}^{x}\left(y-y_{j}\right)^{2}
\end{gathered}
$$

and

$$
\begin{gathered}
\underline{h}^{y}(x, y ; i, j) \stackrel{\text { def }}{=} \\
h_{0,0}^{y}+h_{1,0}^{y}\left(x-x_{i}\right)+h_{0,1}^{y}\left(y-y_{j}\right)+ \\
h_{2,0}^{y}\left(x-x_{i}\right)^{2}+h_{1,2}^{y}\left(x-x_{i}\right)\left(y-y_{j}\right)+h_{0,2}^{y}\left(y-y_{j}\right)^{2} .
\end{gathered}
$$

The coefficients $h_{0,0}^{x}, h_{1,0}^{x}$, etc., are the numerical analogues of $h^{x}\left(x_{i}, y_{j}\right), \frac{\partial h^{2}}{\partial x}\left(x_{i}, y_{j}\right)$, etc., and are unknowns that are to be determined (the $i, j$ subscripts have been omitted for clarity). Generally, the unknown coefficients above are expressed in terms of one or more intermediate variables. This will become clear later, but need not concern us here.

To faithfully represent $\vec{h}$ throughout $\mathcal{D}$, the discrete approximation $\left\{(\vec{h})_{i, j}, i=1, \ldots, N_{i}, j=1, \ldots, N_{j}\right\}$ should: (i) satisfy (2.1) on each cell and each union of cells in $\mathcal{D}$; (ii) have zero divergence; and (iii) satisfy the boundary conditions on $B(\mathcal{D})$.

Let the vertices of the $(i, j)$ th cell be denoted by $P, Q, R$, and $S$ as shown in Figure 3. Then (i) above requires that

$$
\oint_{P Q R S_{i, j}} \underset{\vec{h}}{\vec{d}} \cdot \overrightarrow{d s}=0
$$

where we have taken the integration to be positive in the counterclockwise sense. Let $[J(\overline{P Q})]_{i, j}$ denote the flux of $\vec{h}$ through the line segment $\overline{P Q}_{i, j}$, and similarly for $J(\overline{Q R}), J(\overline{R S})$, and $J(\overline{S P})$. Then, omitting the $i, j$ subscripts, one obtains the flux expressions

$$
J(\overline{P Q})=
$$

$$
\begin{gathered}
\frac{\Delta x^{3}}{12} h_{2,0}^{y}+\Delta x\left[\frac{\Delta y^{2}}{4} h_{0,2}^{y}+\frac{\Delta y}{2} h_{0,1}^{y}+h_{0,0}^{y}\right] \\
J(\overline{Q R})= \\
-\frac{\Delta y^{3}}{12} h_{0,2}^{x}-\Delta y\left[\frac{\Delta x^{2}}{4} h_{2,0}^{x}-\frac{\Delta x}{2} h_{1,0}^{x}+h_{0,0}^{x}\right] \\
-\frac{\Delta x^{3}}{12} h_{2,0}^{y}-\Delta x\left[\frac{\Delta y^{2}}{4} h_{0,2}^{y}-\frac{\Delta y}{2} h_{0,1}^{y}+h_{0,0}^{y}\right] \\
J(\overline{S P})= \\
\frac{\Delta y^{3}}{12} h_{0,2}^{x}+\Delta y\left[\frac{\Delta x^{2}}{4} h_{2,0}^{x}+\frac{\Delta x}{2} h_{1,0}^{x}+h_{0,0}^{x}\right] .
\end{gathered}
$$

Equation (2.10) requires that the fluxes satisfy

$$
J(\overline{P Q})+J(\overline{Q R})+J(\overline{R S})+J(\overline{S P}) \equiv 0 .
$$

Thus, one obtains the fiux conservation constraint

$$
h_{1,0}^{x}+h_{0,1}^{y}=0 .
$$

In view of (2.10), each cell in the mesh is called an conservation element. Since $\overrightarrow{\boldsymbol{h}}$ may be discontinuous across cell interfaces, each cell is also called an solution element. ${ }^{4}$

Imposing condition (2.16), one obtains from (2.11) - (2.14) the following normalized flux expressions:

$$
\begin{gathered}
\frac{J(\overline{P Q})}{\Delta x}= \\
\frac{\Delta x^{2}}{12} h_{2,0}^{y}+\frac{\Delta y^{2}}{4} h_{0,2}^{y}-\frac{\Delta y}{2} h_{1,0}^{x}+h_{0,0}^{y} \\
-\frac{J(\overline{Q R})}{\Delta y}= \\
\frac{\Delta y^{2}}{12} h_{0,2}^{x}+\frac{\Delta x^{2}}{4} h_{2,0}^{x}-\frac{\Delta x}{2} h_{1,0}^{x}+h_{0,0}^{x} \\
-\frac{J(\overline{R S})}{\Delta x}= \\
\frac{\Delta x^{2}}{12} h_{2,0}^{y}+\frac{\Delta y^{2}}{4} h_{0,2}^{y}+\frac{\Delta y}{2} h_{1,0}^{x}+h_{0,0}^{y}
\end{gathered}
$$




$$
\begin{gathered}
\frac{J(\overline{S P})}{\Delta y}= \\
\frac{\Delta y^{2}}{12} h_{0,2}^{x}+\frac{\Delta x^{2}}{4} h_{2,0}^{x}+\frac{\Delta x}{2} h_{1,0}^{x}+h_{0,0}^{x} .
\end{gathered}
$$

Equations (2.16) - (2.20) are sufficient to ensure that (2.1) is satisfied on each cell. To ensure that (2.1) is satisfied globally, it is necessary that fluxes be conserved across cell interfaces.

Let the $(i, j)$ th conservation element be denoted by $C E(i, j)$. Consider the vertical interface between $C E(i, j)$ and $C E(i+1, j)$. The flux leaving $C E(i, j)$ through this interface is given by $[J(\overline{S P})]_{i, j}$. On the other hand, the flux leaving $C E(i+1, j)$ through this same interface is given by $[J(\overline{Q R})]_{i+1, j}$. Flux conservation acroes the interface requires the sum of the two to be zero. Thus, one obtains the interface condition

$$
\begin{aligned}
& {\left[\frac{\Delta y^{2}}{12} h_{0,2}^{x}+\frac{\Delta x^{2}}{4} h_{2,0}^{x}+\frac{\Delta x}{2} h_{1,0}^{x}+h_{0,0}^{x}\right]_{i, j}} \\
& -\left[\frac{\Delta y^{2}}{12} h_{0,2}^{x}+\frac{\Delta x^{2}}{4} h_{2,0}^{x}-\frac{\Delta x}{2} h_{1,0}^{x}+h_{0,0}^{x}\right]_{i+1, j}=0 .
\end{aligned}
$$

Similarly, across horizontal interfaces, one obtains the interface condition

$$
\begin{aligned}
& {\left[\frac{\Delta x^{2}}{12} h_{2,0}^{y}+\frac{\Delta y^{2}}{4} h_{0,2}^{y}-\frac{\Delta y}{2} h_{1,0}^{x}+h_{0,0}^{y}\right]_{i, j}(2.22)} \\
& -\left[\frac{\Delta x^{2}}{12} h_{2,0}^{y}+\frac{\Delta y^{2}}{4} h_{0,2}^{y}+\frac{\Delta y}{2} h_{1,0}^{x}+h_{0,0}^{y}\right]_{i, j+1}=0 .
\end{aligned}
$$

Equations (2.16) - (2.22) above ensure that the integral form of the governing conservation law will be satisfied throughout $\mathcal{D}$.

Having provided for the satisfaction of the more fundamental integral conservation law, we may now turn our attention to the differential conservation law (2.2). Requiring $\vec{h}$ to have zero divergence on each conservation element, one obtains the two secondorder constraints

$$
\begin{aligned}
& 2 h_{2,0}^{x}+h_{1,1}^{y}=0 \\
& 2 h_{0,2}^{y}+h_{1,1}^{x}=0 .
\end{aligned}
$$

By virtue of equations (2.23) - (2.24), the approximate second-order solution $\overrightarrow{\boldsymbol{h}}$ satisfies (2.2) identically, as does the exact second-order solution $\vec{h}_{2}$. In Reference [3] it was rigorously proved that conditions (2.23) -
(2.24) ensure the satisfaction of a necessary condition for the second-order discrete unknowns to converge to their respective analytical counterparts.

We should also emphasize that (2.23) - (2.24) are local conditions, as is the first-order constraint (2.16). Thus, a high-order-accurate simulation of the differential conservation law is possible without stringing together more and more mesh points.

The above approach provides the conceptual framework for the discrete simulation of steady-state flux conservation in two space dimensions. We now demorstrate the applicability of the framework to the Navier-Stokes equations.

\section{Conservation of Mass and Momentum in Incompressible, Viscous Flow}

\section{A. Discrete Flux Vectors}

The governing equations for the conservation of mass and momentum in a steady, two-dimensional, incompressible flow field can be written ${ }^{5}$

$$
\frac{\partial u}{\partial x}+\frac{\partial v}{\partial y}=0
$$

$$
\begin{aligned}
& \frac{\partial}{\partial x}\left(u^{2}+p-\tau_{x x}\right)+\frac{\partial}{\partial y}\left(u v-\tau_{x y}\right)=0 \\
& \frac{\partial}{\partial x}\left(u v-\tau_{x y}\right)+\frac{\partial}{\partial y}\left(v^{2}+p-\tau_{y y}\right)=0
\end{aligned}
$$

where

$$
\begin{gathered}
\tau_{x x}=\frac{2}{3 R e_{L}}\left(2 \frac{\partial u}{\partial x}-\frac{\partial v}{\partial y}\right) \\
\tau_{x y}=\frac{1}{R e_{\iota}}\left(\frac{\partial u}{\partial y}+\frac{\partial v}{\partial x}\right) \\
\tau_{y y}=\frac{2}{3 R e_{L}}\left(2 \frac{\partial v}{\partial y}-\frac{\partial u}{\partial x}\right) .
\end{gathered}
$$

The independent variables $x$ and $y$ are horizontal and vertical Cartesian coordinates, respectively, and $u$ and $v$ dencte their respective velocity components. $p$ denotes the static pressure, and $R e_{L}$ is the Reynolds number, $\frac{\rho U_{\infty} L}{\mu}$, where the viscosity $\mu$ and density $\rho$ are assumed to be constant. $L$ and $U_{\infty}$ refer to some referer ce length and velocity, respectively.

The integral form of (3.1) - (3.3) is given by

$$
\begin{aligned}
& \oint_{S(V)} \vec{h}_{M} \cdot \overrightarrow{d s}=0 \\
& \oint_{S(V)} \vec{h}_{X M} \cdot \overrightarrow{d s}=0
\end{aligned}
$$




$$
\oint_{S(V)} \vec{h}_{Y M} \cdot \overrightarrow{d s}=0
$$

where $\vec{h}_{M}, \vec{h}_{X M}$, and $\vec{h}_{Y M}$ are defined by

$$
\begin{gathered}
\vec{h}_{M} \stackrel{\text { def }}{=}(u, v) \\
\vec{h}_{X M} \stackrel{\text { def }}{=}\left(u^{2}+p-\tau_{x x}, u v-\tau_{x y}\right) \\
\vec{h}_{Y M} \stackrel{\text { def }}{=}\left(u v-\tau_{x y}, v^{2}+p-\tau_{y y}\right) .
\end{gathered}
$$

Equations (3.7) - (3.9) are a coupled system of integral conservation laws whereby mass and momentum are conserved by way of the primitive (intermediate) variables $u, v$, and $p$. The system of equations (3.7) (3.9) is of the same form as system (2.1).

Corresponding to the continuous flux vectors defined by (3.10) - (3.12), we introduce the second-order discrete flux vectors $\vec{\sim}_{M}, \vec{h}_{X M}$, and $\overrightarrow{\underline{h}}_{Y M}$, where

$$
\begin{gathered}
\underline{\vec{h}}_{M} \stackrel{\text { def }}{=}(\underline{u}, \underline{v}) \\
\vec{h}_{X M} \stackrel{\text { def }}{=}\left(\underline{u}^{2}+\underset{\sim}{p}-\tau_{x x}, \underline{u} \underline{v}-\tau_{x y}\right) \\
\overrightarrow{\underline{h}}_{Y M} \stackrel{\text { deff }}{=}\left(\underline{\sim}_{\sim}^{v}-\tau_{x y}, \underline{v}^{2}+\underline{p}-\tau_{y y}\right)
\end{gathered}
$$

and

$$
\begin{gathered}
\underline{\tau}_{x x}=\frac{2}{3 R e_{x}}(2 \partial \underline{u} / \partial x-\partial \underline{v} / \partial y) \\
\tau_{x y}=\frac{1}{R e_{x}}(\partial \underline{u} / \partial y+\partial \underline{v} / \partial x) \\
\tau_{y y}=\frac{2}{3 R e_{z}}(2 \partial \underline{v} / \partial y-\partial \underline{u} / \partial x) .
\end{gathered}
$$

By definition, the discrete primitive variables $\underline{u}, \underline{v}$, and $\underline{p}$ are then second-order expansions which may be written

$$
\begin{gathered}
\underline{u}(x, y ; i, j) \stackrel{\text { def }}{=} \\
u_{0,0}+u_{1,0}\left(x-x_{i}\right)+u_{0,1}\left(y-y_{j}\right)+ \\
u_{2,0}\left(x-x_{i}\right)^{2}+u_{1,1}\left(x-x_{i}\right)\left(y-y_{j}\right)+u_{0,2}\left(y-y_{j}\right)^{2} \\
\underline{v}(x, y ; i, j) \stackrel{\text { def }}{=} \\
v_{0,0}+v_{1,0}\left(x-x_{i}\right)+v_{0,1}\left(y-y_{j}\right)+ \\
v_{2,0}\left(x-x_{i}\right)^{2}+v_{1,1}\left(x-x_{i}\right)\left(y-y_{j}\right)+v_{0,2}\left(y-y_{j}\right)^{2}
\end{gathered}
$$

$p_{2,0}\left(x-x_{i}\right)^{2}+p_{1,1}\left(x-x_{i}\right)\left(y-y_{j}\right)+p_{0,2}\left(y-y_{j}\right)^{2}$.

For clarity, the $i, j$ subscripts have been omitted from the discrete Taylor coefficients $u_{0,0}, v_{0,0}, p_{0,0}$, etc. These coefficients are the unknowns to be solved for.

Each of the discrete flux vectors $\overrightarrow{\underline{h}}_{M}, \vec{h}_{X M}$, and $\vec{h}_{Y M}$ may be expressed in the form of (2.7) - (2.9), where $h_{0,0}^{x}, h_{0,0}^{y}$, etc., are functions of $u_{0,0}, v_{0,0}, p_{0,0}$, $u_{1,0}, v_{1,0}, p_{1,0}$, etc. For example, in reference to $\overrightarrow{\underline{h}}=$ $\vec{h}_{X M}, h_{0,0}^{x}$ is the constant term of the expression $\underline{\underline{u}}^{2}+$ $p-\tau_{x x}$, which is given by $u_{0,0}^{2}+p_{0,0}-\frac{2}{3 R e_{1}}\left(2 u_{1,0}-v_{0,1}\right)$. In the Appendix, we present the functional expressions for each component of the discrete flux vectors $\vec{\sim}_{M}$, $\vec{h}_{X M}$, and $\vec{h}_{Y M}$.

\section{B. Constant-Pressure-Gradient Flows}

\section{Laminar Flow in a Straight Channel}

Consider the channel geometry and associated mesh shown in Figure 4. On each solution element, we represent $u, v$, and $p$ through the second-order expansions (3.19) - (3.21). There are 18 discrete unknowns on each cell. Since there are $18 N_{i} N_{j}$ unknowns altogether, $18 N_{i} N_{j}$ conditions are required to have a closed system of equations. Thus, for the present equilibrium-type boundary value problem, closing the system requires a global matching of numbers of conditions and unknowns.

The conditions to be imposed are obtained from Section II. For each of the three conservation laws (3.1) - (3.3), we must impose the local constraints (2.16), (2.23) and (2.24) on each conservation element. This provides $9 N_{i} N_{j}$ conditions. (See equations (A.1) - (A.9) in the Appendix.) The interface conditions (2.21) and (2.22) must also be satisfied. This provides an additional $3 N_{j}\left(N_{i}-1\right)+3 N_{i}\left(N_{j}-1\right)=$ $6 N_{i} N_{j}-3 N_{i}-3 N_{j}$ conditions.

Another $4 N_{i}+3 N_{j}$ conditions are obtained by way of boundary conditions. At the channel inlet, we specify the two components of velocity, and at the exit we specify the pressure. Along the upper and lower walls we require that there be zero mass flux. In addition, we impose the no slip condition for $u$ at the midpoint of the wall face of each cell.

For laminar flow in a straight channel, it is well known that the streamwise pressure gradient is nearly constant, except in a short entrance region. Thus, 
$\frac{\partial^{2} p}{\partial x^{2}} \approx 0$ and $\frac{\partial^{2} p}{\partial x \partial y} \approx 0$ throughout most of the channel. It is reasonable then to make the simplifying assumption that $p_{2,0}=0$ and $p_{1,2}=0$ on each cell. This provides an additional $2 N_{i} N_{j}$ conditions.

The number of conditions still needed to close the system is then $N_{i}\left(N_{j}-1\right)$. Since this is the number of horizontal interfaces in the mesh, there is an additional degree of freedom in specifying horizontal interface conditions. We choose to close the system by requiring the $u$ velocity to be continuous at the midpoint of each horizontal interface.

The discrete boundary value problem outlined above is a coupled system of second-order polynomial equations in the unknowns $u_{0,0}, v_{0,0}, p_{0,0}$, etc. However, six of the higher-order unknowns can be easily eliminated from the system using the local constraints (A.1) - (A.9). (See equations (A.10) - (A.15).) The total number of unknowns that must be explicitly solved for is thus reduced from $16 N_{i} N_{j}$ to $10 N_{i} N_{j}$. Of the equations that remain, $3 N_{i} N_{j}$ represent local conditions, and the remaining $7 N_{i} N_{j}$ represent interface and boundary conditions. Numerical solutions to the nonlinear system of equations may be obtained by Newton's method. ${ }^{1}$ The Jacobian matrix has the form shown in Figure 5.

In Figures 6 and 7 we present numerical results from calculations performed at Reynolds numbers (based on channel height $h$ ) of 100 and 2000 . The specified inlet velocity profile for each case is $u_{i}=\frac{7}{6}\left[1-\left[2\left(y-\frac{1}{2}\right)\right]^{6}\right]$ (following Dill and Himansu ${ }^{8}$ ). Along with the numerical results, we also show the inlet profile and fully developed analytical solution. The $R e=100$ results were obtained from the $39 \times 6$ grid shown in Figure 4. Due to the thinner boundary layer, the $R e=2000$ results were obtained from a $39 \times 10$ mesh. For each case, Newton's method converged to a maximum residual error of $10^{-10}$ in four and five iterations, respectively (starting from uniform flow). The corresponding CPU times on a Cray YMP were 1.1 and 3.5 seconds.

\section{High-Reynolds-Number Flow} Around a Finite Flat Plate

In Figure 8 we show a typical mesh for calculation of the thin airfoil boundary layer problem. The airfoil lies on the $x$-axis between 0 and 1 , and the mesh includes both an upstream and wake region. The grid spacing is exponentially stretched in the $y$ direction, and the spacing may also be nonuniform in the $x$ direction.

As in the channel flow problem, there are a total of $18 N_{i} N_{j}$ unknowns to be solved for. The methodology for clcsing the system here closely follows that given in the previous section.

Lat $N_{a}$ denote the number of solution elements between the airfoil leading and trailing edges. Then (2.16) and (2.21) - (2.24) provide $9 N_{i} N_{j}+3 N_{j}\left(N_{i}-\right.$ 1) $+3 N_{i}\left(N_{j}-1\right)-3 N_{a}=15 N_{i} N_{j}-3 N_{i}-3 N_{j}-3 N_{a}$ conditions.

Boundary conditions provide an additional $4 N_{i}+$ $3 N_{j}+4 N_{a}$ conditions. For each cell adjacent to the airfoil, we require the mass flux through the wall face, and the $u$ velocity at the midpoint of the wall face, to be zero. At the upstream boundary, we specify the velocity, and downstream we specify the pressure. Along the free-stream boundary cells, we specify zero $y$ gradient conditions for $u$ and $v$. Imposing the zero pressure gradient condition, we set $p_{2,0}=0$ and $p_{1,1}=0$ on each cell, for an additional $2 N_{i} N_{j}$ condtions. Finally, an additional $N_{i}\left(N_{j}-1\right)-N_{a}$ conditions are obtained by requiring the $u$ velocity to be continuous at the midpoint of horizontal cell interfaces.

In Figure 9 we compare the predicted boundary layer profile with the Blasius solution at four different locations along the airfoil. The Reynolds number based on airfoil chord is 100,000 . The results were obtained from an $81 \times 20$ grid with uniform $x$ spacing and nonuniform $y$ spacing with $12.5 \%$ exponential stretching. Only ten cells are used across the boundary layer, since the flow field is computed on each side of the airfoil. The $y$ spacing at the wall was $\Delta y_{w}=.0015$, and the free stream boundaries were located at $y= \pm .027$. The CPU time required on a Cray YMP was 50.7 seconds (nine Newton iterations), with uniform flow as the starting solution.

Figure 10 compares the predicted $v$ velocity at $x$ $=.805$ with the Blasius $v$ velocity. The differences that appear near the edge of the boundary layer are believed to be due to finite airfoil effects $[7$, p. 137].

In Figure 11 we show the pressure coefficient in the trailing edge region. The results were computed on an $110 \times 22$ grid that was refined near the trailing edge with exponential $x$ stretching. The $x$ spacing at the trailing edge was .007 . Our results show the pressure coefficient to have a minimum value of -.014 , which agrees well with the Reduced Navier-Stokes (RNS) calculations of Srinivasan and Rubin. ${ }^{6}$

\section{General Flow Fields}

\section{Generalized Closure Procedure}

For a general two-dimensional problem with an $N_{i}$ $x N_{j}$ mesh, the local expansions (3.19) - (3.21) provide a total of $18 N_{i} N_{j}$ unknowns. Thus, $18 N_{i} N_{j}$ conditions are always required to close the discrete system of 
equations. In Section II. B., we imposed $16 N_{i} N_{j}$ general conditions, and then added the $2 N_{i} N_{j}$ special conditions $\left[p_{2,0}\right]_{i, j}=0,\left[p_{1,1}\right]_{i, j}=0$. Since the general conditions already ensure that the integral and differential forms of the governing conservation laws are fully satisfied, it will be necessary to introduce higher-order conditions to close the system. Such higher-order conditions can be imposed either on the discrete primitive variables, or on the discrete flux vectors. Due to the more fundamental nature of the flux vectors, the second alternative is generally preferred over the first.

We are thus led to consider the first-order dipole moment interface conditions

$$
\int_{x_{1}}^{x_{2}}\left(x-x_{i}\right)\left(\vec{h}_{i, j}-\vec{h}_{i, j+1}\right) \cdot \overrightarrow{d s}=0
$$

and

$$
\int_{y_{2}}^{y_{2}}\left(y-y_{j}\right)\left(\vec{h}_{i, j}-\vec{h}_{i+1, j}\right) \cdot \overrightarrow{d s}=0,
$$

where $x_{1}$ and $x_{2}$ denote the endpoints of an horizontal interface between $C E(i, j)$ and $C E(i, j+1)$, and $y_{1}$ and $y_{2}$ denote the endpoints of an vertical interface between $C E(i, j)$ and $C E(i+1, j)$. Since aerodynamic flow fields are generally dominated by gradients in the cross-flow direction, equation (3.22) will usually be preferred over equation (3.23). However, both conditions are fully justifiable, since the exact solution $\vec{h}$ satisfies both equations.

For a rectangular grid, equations (3.22) and (3.23) become

$$
\left[h_{1,0}^{y}+\frac{\Delta y}{2} h_{1,1}^{y}\right]_{i, j}-\left[h_{1,0}^{y}-\frac{\Delta y}{2} h_{1,1}^{y}\right]_{i, j+1}=0
$$

and

$$
\left[h_{0,1}^{x}+\frac{\Delta x}{2} h_{1,1}^{x}\right]_{i, j}-\left[h_{0,1}^{x}-\frac{\Delta x}{2} h_{1,1}^{x}\right]_{i+1, j}=0,
$$

respectively.

We now consider again the channel and flat plate flow fields. For channel flow, we impose (3.24) for $\overrightarrow{\vec{h}}=\vec{\sim}_{X M}$ and $\underset{\boldsymbol{h}}{\vec{h}}=\overrightarrow{\underline{h}}_{Y M}$, thereby obtaining an additional $2 N_{i}\left(N_{j}-1\right)$ conditions. The final $2 N_{i}$ conditions may be obtained by imposing an additional boundary condition on the upper and lower walls. From the $y$ momentum equation one obtains the pressure boundary condition ${ }^{5}$

$$
\frac{\partial p}{\partial y}=\frac{1}{R e} \frac{\partial^{2} v}{\partial y^{2}}
$$

which may be imposed at the center point of the wall face of each cell. (Equation (3.26) is not a boundary condition in the usual sense, but is instead a differential equation to be satisfied on a wall parallel to the $x$-axis. Even though we already require the $y$ momentum equation to be satisfied identically on each cell, equation (3.26) still represents an independent condition which may be imposed along the wall.)

For the flat plate problem, imposing (3.24) for the $x$ - and $y$-momentum equations provides $2 N_{i}\left(N_{j}-1\right)$ $2 N_{a}$ conditions, where $N_{a}$ is the number of cells between the leading and trailing edges. Applying (3.26) to each side of the airfoil gives $2 N_{a}$ conditions, and imposing the zero pressure gradient condition as a freestream boundary condition provides the final $2 N_{i}$ conditions.

In general, conditions (3.22) - (3.23), together with additional boundary conditions, can be used to close the discrete system of equations for an arbitrary flow field. By introducing such higher-order conditions, the system is not only closed, but additional physics are brought into the scheme. Thus, it is possible to adapt one's particular choice of closure to the physics of a specific flow field.

In the general case, there are an additional $2 N_{i} N_{j}$ unknowns to be solved for. However, the size of the system to be solved is still $10 N_{i} N_{j}$, as in the constant-pressure-gradient scheme. The reason is that, in the general case, the two new unknowns $p_{2,0}$ and $p_{1,1}$ can be explicitly eliminated from the system. (See equations (A.20) - (A.21).) Thus, a total of $8 N_{i} N_{j}$ unknowns are eliminated, and $10 N_{i} N_{j}$ still remain.

Application of the generalized scheme described above to the channel flow problems of Figures 6 and 7 reproduces the earlier results. The comparison is shown in Figures 12 - 13.

For flat plate flow, the neglected terms $p_{2,0}$ and $p_{1,1}$ become significant for Reynolds numbers less than about 7500 . Figures 14 and 15 compare results from the two schemes at Reynolds numbers of 7500 and 1000 . As Re decreases, the boundary layer becomes much thicker and the pressure gradient varies significantly throughout the flow field. At the lower Reynolds number, the improved accuracy of the general scheme over the constant-pressure-gradient scheme is readily apparent.

\section{Extension to Nonorthogonal Cartesian Grids}

Extension of the generalized scheme presented above to nonorthogonal grids can be accomplished in a straightforward manner. Consider the nonorthogonal mesh shown in Figure 16. We denote the mesh points 
by $\left\{\left(x_{i, j}, y_{i, j}\right), i=0, \ldots, N_{i}, j=0, \ldots, N_{j}\right\}$ and the center points by $\left\{\left(x_{c i, j}, y_{c i, j}\right), i=1, \ldots, N_{i}, j=1, \ldots, N_{j}\right\}$, where $x_{c i, j} \stackrel{\text { def }}{=}\left(x_{i-1, j-1}+x_{i, j-1}+x_{i, j}+x_{i-1, j}\right) / 4$ and $y_{c i, j} \stackrel{\text { def }}{=}\left(y_{i-1, j-1}+y_{i, j-1}+y_{i, j}+y_{i-1, j}\right) / 4$. On each solution element, the discrete flux vectors and primitive variables are expanded about the cell center $\left(x_{c i, j}, y_{c i, j}\right)$.

Since we require $\vec{h}_{M}, \overrightarrow{\underline{h}}_{X M}$, and $\overrightarrow{\underline{h}}_{Y M}$ to be identical solutions of the differential conservation law (2.2), local flux conservation on each $C E(i, j)$ is assured. This follows from the divergence theorem. Consequently, to guarantee global flux conservation, we need only ensure that fluxes are conserved across the nonorthogonal cell interfaces. This in turn requires that the normalized flux expressions (2.17) - $(2.20)$ be generalized to account for the nonorthogonality of the mesh.

The generalized fluxes are derived most easily using a local coordinate system relative to the cell center (see Figure 17). The cell vertices $P, Q, R, S$, relative to $\left(x_{c i, j}, y_{c i, j}\right)$, are given by $\left(\frac{\Delta x_{2}}{2}, \frac{\Delta y_{2}}{2}\right),\left(-\frac{\Delta x_{i}}{2}, \frac{\Delta y_{i}}{2}\right)$, $\left(-\frac{\Delta x_{x}}{2},-\frac{\Delta y_{r}}{2}\right),\left(\frac{\Delta x_{k}}{2},-\frac{\Delta y_{l}}{2}\right)$, where

$$
\begin{gathered}
\frac{\Delta x_{p}}{2} \stackrel{\text { def }}{=} x_{i, j}-x_{c i, j} \\
\frac{\Delta y_{p}}{2} \stackrel{\text { def }}{=} y_{i, j}-y_{c i, j} \\
\frac{\Delta x_{q}}{2} \stackrel{\text { def }}{=} x_{c i, j}-x_{i-1, j} \\
\frac{\Delta y_{q}}{2} \stackrel{\text { def }}{=} y_{i-1, j}-y_{c i, j} \\
\frac{\Delta x_{r}}{2} \stackrel{\text { def }}{=} x_{c i, j}-x_{i-1, j-1} \\
\frac{\Delta y_{r}}{2} \stackrel{\text { def }}{=} y_{c i, j}-y_{i-1, j-1} \\
\frac{\Delta x_{s}}{2} \stackrel{\text { def }}{=} x_{i, j-1}-x_{c i, j} \\
\frac{\Delta y_{j}}{2} \stackrel{\text { def }}{=} y_{c i, j}-y_{i, j-1} .
\end{gathered}
$$

We assume that $\frac{\Delta x_{2}}{2}, \frac{\Delta y_{k}}{2}, \frac{\Delta x_{2}}{2}$, etc., are all positive.

For the flux through an $\overline{P Q}$ or $\overline{R S}$ interface, let

$$
\begin{array}{r}
C_{n-k, k}=h_{n-k, k}^{y}-m h_{n-k, k}^{x} \\
\quad \text { for } n=0,1,2, k=0, \ldots, n,
\end{array}
$$

where $m$ will be defined shortly. Then

$$
\frac{J(\overline{P Q})}{\frac{\Delta x_{Y}}{2}+\frac{\Delta x_{q}}{2}}=C_{0,0}+\delta C_{0,1}+\delta^{2} C_{0,2}
$$

$$
\begin{gathered}
+\Delta^{1}\left(C_{1,0}+m C_{0,1}+\delta C_{1,1}+2 m \delta C_{0,2}\right) \\
+\Delta^{2}\left(C_{2,0}+m C_{1,1}+m^{2} C_{0,2}\right)
\end{gathered}
$$

where

$$
\begin{gathered}
m \stackrel{\text { def }}{=}\left(\frac{\Delta y_{p}}{2}-\frac{\Delta y_{q}}{2}\right) /\left(\frac{\Delta x_{p}}{2}+\frac{\Delta x_{q}}{2}\right) \\
\delta \stackrel{\text { def }}{=} \frac{\Delta y_{q}}{2}+m \frac{\Delta x_{q}}{2} \\
\Delta^{1} \stackrel{\text { def }}{=} \frac{1}{2}\left(\frac{\Delta x_{p}}{2}-\frac{\Delta x_{q}}{2}\right)
\end{gathered}
$$

and

$$
\begin{gathered}
\Delta^{2} \stackrel{\text { def }}{=} \frac{1}{3}\left[\left(\frac{\Delta x_{p}}{2}\right)^{2}-\frac{\Delta x_{p}}{2} \frac{\Delta x_{q}}{2}+\left(\frac{\Delta x_{q}}{2}\right)^{2}\right] \\
-\frac{J(\overline{R S})}{\frac{\Delta x_{x}}{2}+\frac{\Delta x_{q}}{2}}=C_{0,0}+\delta C_{0,1}+\delta^{2} C_{0,2} \\
+\Delta^{1}\left(C_{1,0}+m C_{0,1}+\delta C_{1,1}+2 m \delta C_{0,2}\right) \\
+\Delta^{2}\left(C_{2,0}+m C_{1,1}+m^{2} C_{0,2}\right)
\end{gathered}
$$

where

$$
\begin{array}{r}
m \stackrel{\text { def }}{=}\left(\frac{\Delta y_{\tau}}{2}-\frac{\Delta y_{s}}{2}\right) /\left(\frac{\Delta x_{r}}{2}+\frac{\Delta x_{s}}{2}\right) \\
\delta \stackrel{\text { def }}{=}-\frac{\Delta y_{r}}{2}+m \frac{\Delta x_{r}}{2} \\
\Delta^{1} \stackrel{\text { def }}{=} \frac{1}{2}\left(\frac{\Delta x_{s}}{2}-\frac{\Delta x_{r}}{2}\right)
\end{array}
$$

and

$$
\Delta^{2} \stackrel{\text { def }}{=} \frac{1}{3}\left[\left(\frac{\Delta x_{r}}{2}\right)^{2}-\frac{\Delta x_{r}}{2} \frac{\Delta x_{s}}{2}+\left(\frac{\Delta x_{s}}{2}\right)^{2}\right]
$$

The normalized first-order dipole moment flux through an $\overline{P Q}$ interface is

$$
\begin{gathered}
{\left[\frac{J(\overline{P Q})}{\left.\frac{\Delta x_{x}}{2}+\frac{\Delta x_{q}}{2}\right]_{d p}=}\right.} \\
\Delta^{1}\left(C_{0,0}+\delta C_{0,1}+\delta^{2} C_{0,2}\right) \\
+\Delta^{2}\left(C_{1,0}+m C_{0,1}+\delta C_{1,1}+2 m \delta C_{0,2}\right) \\
+\Delta^{3}\left(C_{2,0}+m C_{1,1}+m^{2} C_{0,2}\right)
\end{gathered}
$$

where 
$\Delta^{3} \stackrel{\text { def }}{=}$
$\frac{1}{4}\left[\left(\frac{\Delta x_{p}}{2}\right)^{3}-\left(\frac{\Delta x_{p}}{2}\right)^{2} \frac{\Delta x_{q}}{2}+\frac{\Delta x_{p}}{2}\left(\frac{\Delta x_{q}}{2}\right)^{2}-\left(\frac{\Delta x_{q}}{2}\right)^{3}\right]$, and all other parameters are defined as in (3.29a) (3.29d). Similarly, the normalized first-order dipole moment flux through an $\overline{R S}$ interface is

$$
\begin{gathered}
-\left[\frac{J(\overline{R S})}{\left.\frac{\Delta x_{x}}{2}+\frac{\Delta x_{x}}{2}\right]_{d p}}=\right. \\
\Delta^{1}\left(C_{0,0}+\delta C_{0,1}+\delta^{2} C_{0,2}\right) \\
+\Delta^{2}\left(C_{1,0}+m C_{0,1}+\delta C_{1,1}+2 m \delta C_{0,2}\right) \\
+\Delta^{3}\left(C_{2,0}+m C_{1,1}+m^{2} C_{0,2}\right)
\end{gathered}
$$

where

$$
\Delta^{3} \stackrel{\text { def }}{=}
$$

$\frac{1}{4}\left[\left(\frac{\Delta x_{s}}{2}\right)^{3}-\left(\frac{\Delta x_{s}}{2}\right)^{2} \frac{\Delta x_{r}}{2}+\frac{\Delta x_{s}}{2}\left(\frac{\Delta x_{r}}{2}\right)^{2}-\left(\frac{\Delta x_{r}}{2}\right)^{3}\right]$, and all other parameters are defined as in (3.30a) (3.30d).

For the flux through an $\overline{Q R}$ or $\overline{S P}$ interface, let

$$
\begin{array}{r}
C_{n-k, k}=h_{n-k, k}^{x}-m h_{n-k, k}^{y} \\
\quad \text { for } n=0,1,2, k=0, \ldots, n .
\end{array}
$$

Then similar to the above, we have

$$
\begin{gathered}
-\frac{J(\overline{Q R})}{\frac{\Delta y_{q}}{2}+\frac{\Delta y_{x}}{2}}=C_{0,0}+\delta C_{1,0}+\delta^{2} C_{2,0} \\
+\Delta^{1}\left(C_{0,1}+m C_{1,0}+\delta C_{1,1}+2 m \delta C_{2,0}\right) \\
+\Delta^{2}\left(C_{0,2}+m C_{1,1}+m^{2} C_{2,0}\right)
\end{gathered}
$$

where

$$
\begin{gathered}
m \stackrel{\text { def }}{=}\left(\frac{\Delta x_{r}}{2}-\frac{\Delta x_{q}}{2}\right) /\left(\frac{\Delta y_{r}}{2}+\frac{\Delta y_{q}}{2}\right) \\
\delta \stackrel{\text { def }}{=}-\frac{\Delta x_{r}}{2}+m \frac{\Delta y_{r}}{2} \\
\Delta^{1} \stackrel{\text { def }}{=} \frac{1}{2}\left(\frac{\Delta y_{q}}{2}-\frac{\Delta y_{r}}{2}\right)
\end{gathered}
$$

and

$$
\Delta^{2} \stackrel{\text { def }}{=} \frac{1}{3}\left[\left(\frac{\Delta y_{q}}{2}\right)^{2}-\frac{\Delta y_{q}}{2} \frac{\Delta y_{r}}{2}+\left(\frac{\Delta y_{r}}{2}\right)^{2}\right]
$$

$$
\begin{aligned}
& \frac{J(\overline{S P})}{\frac{\Delta y_{x}}{2}+\frac{\Delta y_{x}}{2}}=C_{0,0}+\delta C_{1,0}+\delta^{2} C_{2,0} \\
& +\Delta^{1}\left(C_{0,1}+m C_{1,0}+\delta C_{1,1}+2 m \delta C_{2,0}\right) \\
& +\Delta^{2}\left(C_{0,2}+m C_{1,1}+m^{2} C_{2,0}\right)
\end{aligned}
$$

where

$$
\begin{gathered}
m \stackrel{\text { def }}{=}\left(\frac{\Delta x_{p}}{2}-\frac{\Delta x_{s}}{2}\right) /\left(\frac{\Delta y_{p}}{2}+\frac{\Delta y_{s}}{2}\right) \\
\delta \stackrel{\text { def }}{=} \frac{\Delta x_{s}}{2}+m \frac{\Delta y_{s}}{2} \\
\Delta^{1} \stackrel{\text { def }}{=} \frac{1}{2}\left(\frac{\Delta y_{p}}{2}-\frac{\Delta y_{s}}{2}\right)
\end{gathered}
$$

and

$$
\Delta^{2} \stackrel{\text { def }}{=} \frac{1}{3}\left[\left(\frac{\Delta y_{p}}{2}\right)^{2}-\frac{\Delta y_{p}}{2} \frac{\Delta y_{s}}{2}+\left(\frac{\Delta y_{s}}{2}\right)^{2}\right]
$$

The normalized first-order dipole moment flux through an $\overline{Q R}$ interface is

$$
\begin{gathered}
-\left[\frac{J(\overline{Q R})}{\frac{\Delta y_{y}}{2}+\frac{\Delta y_{x}}{2}}\right]_{d p}= \\
\Delta^{1}\left(C_{0,0}+\delta C_{1,0}+\delta^{2} C_{2,0}\right) \\
+\Delta^{2}\left(C_{0,1}+m C_{1,0}+\delta C_{1,1}+2 m \delta C_{2,0}\right) \\
+\Delta^{3}\left(C_{0,2}+m C_{1,1}+m^{2} C_{2,0}\right)
\end{gathered}
$$

where

$$
\Delta^{3} \stackrel{\text { def }}{=}
$$

$\frac{1}{4}\left[\left(\frac{\Delta y_{q}}{2}\right)^{3}-\left(\frac{\Delta y_{q}}{2}\right)^{2} \frac{\Delta y_{r}}{2}+\frac{\Delta y_{q}}{2}\left(\frac{\Delta y_{r}}{2}\right)^{2}-\left(\frac{\Delta y_{r}}{2}\right)^{3}\right]$, and the remaining parameters are defined by (3.34a) (3.34d).

Finally, the normalized first-order dipole moment flux through an $\overline{S P}$ interface is

$$
\begin{gathered}
{\left[\frac{J(\overline{S P})}{\frac{\Delta y_{p}}{2}+\frac{\Delta y_{p}}{2}}\right]_{d p}=} \\
\Delta^{1}\left(C_{0,0}+\delta C_{1,0}+\delta^{2} C_{2,0}\right) \\
+\Delta^{2}\left(C_{0,1}+m C_{1,0}+\delta C_{1,1}+2 m \delta C_{2,0}\right) \\
+\Delta^{3}\left(C_{0,2}+m C_{1,1}+m^{2} C_{2,0}\right)
\end{gathered}
$$


where

$$
\Delta^{3} \stackrel{\text { def }}{=}
$$

$\frac{1}{4}\left[\left(\frac{\Delta y_{p}}{2}\right)^{3}-\left(\frac{\Delta y_{p}}{2}\right)^{2} \frac{\Delta y_{b}}{2}+\frac{\Delta y_{p}}{2}\left(\frac{\Delta y_{b}}{2}\right)^{2}-\left(\frac{\Delta y_{b}}{2}\right)^{3}\right]$,

and the remaining parameters are defined by (3.35a) (3.35d).

To complete the nonorthogonal formulation, one must modify the pressure boundary condition (3.26) to account for any variation in the wall slope. The remaining boundary conditions require no special treatment.

In Figures 18 - 20 we consider channel flow with a converging or diverging section. For each case, the ramp length is one channel height, and the inlet velocity is $u_{i}=\frac{7}{6}\left[1-\left[2\left(y-\frac{1}{2}\right)\right]^{6}\right]$. The ramp angles for the three cases are 10,15 , and 5 degrees, respectively. In Figures $18 \mathrm{~b}$. and $20 \mathrm{~b}$., we consider flow at a Reynolds number of 100 . The calculations were run on the $32 \times 8$ grids shown in Figures 18 a. and 20 a. The two cases converged in six and eight Newton iterations, respectively. For both cases, the results indicate that the flow becomes fully developed by the end of the channel. In Figure 19 b., results are presented for flow at a Reynolds number of 200 . The calculations were performed on the $32 \times 10$ grid shown in Figure 19 a. For this nonsymmetrical flow at a higher Reynolds number, Newton's method required ten iterations to converge (11.0 seconds on a Cray YMP). Unlike the results in Figures $18 \mathrm{~b}$. and $20 \mathrm{~b}$., the flow does not become fully developed by the end of the channel. This is consistent with the theory for developing laminar flow in a channel $[7$, p. 186].

A similar calculation to the above, using a general coordinates STS implicit scheme, has been carried out by Dill and Himansu. ${ }^{8}$

\section{Accuracy of Derivatives}

The numerical results presented thus far have demonstrated that the current method can resolve developing boundary layers on coarse grids. This clearly indicates that the discrete derivatives, at least through some order, are being obtained accurately. The accuracy of the discrete derivatives is a current research topic, and will be discussed in detail in a future paper.

Our experience to date indicates that the accuracy of the first-order derivatives is in general very good. On the other hand, the accuracy of the second-order derivatives varies considerably, and appears to depend on a number of factors.

As a model problem for estimating the accuracy of the derivatives, we consider the flow field around a finite flat plate. The Blasius similarity solution provides the first-order asymptotic solution to the NavierStokes equations which is valid near the wall and away from the leading and trailing edges. 9,10 Thus, at very high Reynolds numbers, the Blasius solution can be used to obtain analytical values for the derivatives of $u$ and $v$.

In Figure 22, we compare the predicted $\frac{\partial u}{\partial y}$ (i.e., $u_{0,1}$ ) with the Blasius $\frac{\partial u}{\partial y}$. There is in general very good agreement between the two. (The numerical issues involved in obtaining accurate derivatives of the Blasius solution are addressed in a paper which is currently being written. ${ }^{11}$ ) The large value of $\frac{\partial u}{\partial y}$ near the wall is also evident.

Figure 23 compares the predicted $\frac{\partial^{2} u}{\partial y^{2}}$ (i.e., $2 u_{0,2}$ ) with the Blasius $\frac{\partial^{2} u}{\partial y^{2}}$. Although not as accurate as $u_{0,1}$, the relative error of $u_{0,2}$ is of the order of $10^{-3}$ at a number of grid points. Note that the magnitude of $\frac{\partial^{2} u}{\partial y^{2}}$ is greater than $10^{3}$ throughout most of the boundary layer, and obtains a maximum value which is greater than $10^{4}$.

\section{Space-Time Flux Conservation for the Unsteady Navier-Stokes Equations}

The discrete formulation presented above for the steady, incompressible Navier-Stokes equations is designed and constructed to simulate steady-state boundary value problems which are of equilibrium type. However, when considering the unsteady NavierStokes equations, one is confronted with an initialboundary value problem which is of a marching type. Thus, the problem of space-time flux conservation essentially reduces to how to march the discrete solution forward in time in such a way that space-time flux conservation is always satisfied. In general, one may advanise the solution in an explicit or implicit manner. In either case, the focus of the current method is to ensure that fluxes are conserved in space-time, through the use of Taylor series expansions that identically satisfy the governing integral and differential conservation laws.

As a model problem for the unsteady NavierStokes equations, Chang ${ }^{2}$ considered the shock tube problem with viscosity. The governing equations are the 1-D, unsteady Navier-Stokes equations. Let $\rho, v$, $p$, and $\gamma$ be the mass density, velocity, pressure, and constant specific heat ratio, respectively. Let

$$
u_{1}=\rho
$$




$$
\begin{gathered}
u_{2}=\rho v \\
u_{3}=p /(\gamma-1)+(1 / 2) \rho v^{2} \\
f_{1}=u_{2} \\
f_{2}=(\gamma-1) u_{3}+(1 / 2)(3-\gamma)\left(u_{2}\right)^{2} / u_{1} \\
f_{3}=\gamma u_{2} u_{3} / u_{1}-(1 / 2)(\gamma-1)\left(u_{2}\right)^{3} /\left(u_{1}\right)^{2}
\end{gathered}
$$

and let

$$
\begin{gathered}
\tilde{f}_{1}=0 \\
\tilde{f}_{2}=\frac{4}{3 \operatorname{Re}} \frac{u_{2}}{u_{1}} \\
\tilde{f}_{3}=\frac{2}{3 \operatorname{Re}}\left(\frac{u_{2}}{u_{1}}\right)^{2}+\frac{\gamma}{\operatorname{RePr}}\left[\frac{u_{3}}{u_{1}}-\frac{\left(u_{2}\right)^{2}}{2\left(u_{1}\right)^{2}}\right],
\end{gathered}
$$

where Re and $\operatorname{Pr}$ denote the Reynolds number and Prandtl number, respectively. Then the governing equations may be written ${ }^{2}$

$$
\frac{\partial u_{m}}{\partial t}+\frac{\partial f_{m}}{\partial x}-\frac{\partial^{2} \tilde{f}_{m}}{\partial x^{2}}=0, \quad m=1,2,3,
$$

or

$$
\vec{\nabla} \cdot \vec{h}_{m}=0
$$

where

$$
\vec{\nabla} \stackrel{\text { def }}{=}\left(\frac{\partial}{\partial x}, \frac{\partial}{\partial t}\right)
$$

and

$$
\vec{h}_{m}=\left(f_{m}-\frac{\partial \tilde{f}_{m}}{\partial x}, u_{m}\right), \quad m=1,2,3 .
$$

The integral form of (4.11) is then

$$
\oint_{s(V)} \vec{h}_{m} \cdot \overrightarrow{d s}=0
$$

where $V$ is an arbitrary region in the space-time $E_{2}$.

Let $E_{2}$ be discretized by solution elements $S E(j, n)$ and conservation elements $C E(j, n)$ of Type $I$, as described in [2] and [12]. Then, associated with each $S E(j, n)$, let $u_{m}, f_{m}$ and $\tilde{f}_{m}$ be represented in discrete form through the linear expansions

$$
\begin{gathered}
u_{m}^{*}(x, t ; j, n) \stackrel{\text { def }}{=} \\
\left(u_{m}\right)_{j}^{n}+\left(u_{m x}\right)_{j}^{n}\left(x-x_{j}\right)+\left(u_{m t}\right)_{j}^{n}\left(t-t^{n}\right) \\
f_{m}^{*}(x, t ; j, n) \stackrel{\text { def }}{=} \\
\left(f_{m}\right)_{j}^{n}+\left(f_{m x}\right)_{j}^{n}\left(x-x_{j}\right)+\left(f_{m t}\right)_{j}^{n}\left(t-t^{n}\right) \\
\tilde{f}_{m}^{*}(x, t ; j, n) \stackrel{\text { def }}{=}
\end{gathered}
$$

$$
\begin{gathered}
\left(\tilde{f}_{m}\right)_{j}^{n}+\left(\tilde{f}_{m x}\right)_{j}^{n}\left(x-x_{j}\right)+\left(\tilde{f}_{m t}\right)_{j}^{n}\left(t-t^{n}\right) \\
m=1,2,3 .
\end{gathered}
$$

The discrete coefficients $\left(u_{m}\right)_{j}^{n},\left(u_{m x}\right)_{j}^{n}$, and $\left(u_{m t}\right)_{j}^{n}, m=1,2,3$, represent nine unknowns associated with each $S E(j, n)$. The coefficients $\left(f_{m}\right)_{j}^{n},\left(\tilde{f}_{m}\right)_{j}^{n}$, etc., are known functions of the $\left(u_{m}\right)_{j}^{n},\left(u_{m x}\right)_{j}^{n}$, and $\left(u_{m t}\right)_{j}^{n}$. By requiring $\vec{h}_{m}^{*} \stackrel{\text { def }}{=}\left(f_{m}^{*}-\frac{\partial \bar{j}_{m}^{*}}{\partial x}, u_{m}^{*}\right), m=$ $1,2,3$, to satisfy $(4.11)$, each $\left(u_{m t}\right)_{j}^{n}$ may also be expressed as a known function of the $\left(u_{m}\right)_{j}^{n}$ and $\left(u_{m x}\right)_{j}^{n}$. Thus, the marching problem from one time level to the next may be expressed entirely in terms of the discrete unknowns $\left(u_{m}\right)_{j}^{n}$ and $\left(u_{m x}\right)_{j}^{n}$. The marching conditions for $\left(u_{m}\right)_{j}^{n}$ and $\left(u_{m x}\right)_{j}^{n}$ are obtained by requiring $\vec{h}_{m}^{*}$ to satisfy

$$
\oint_{S(C E(j, n)} \vec{h}_{m}^{*} \cdot \overrightarrow{d s}=0
$$

for all $(j, n)$. The reader is referred to Reference [2] for the details.

In Figure 23, numerical solutions are presented to the shock tube problem with viscosity at a Reynolds number of 12,000 . Note that the shock is resolved in a single mesh point, and that the contact discontinuity is spread over only three mesh points. No flux limiters, weighting functions, or other ad hoc parameters were used in the calculation.

\section{Summary}

A new numerical method is being developed for solving the Navier-Stokes equations. In this paper, the authors' 2-D, steady-state implicit scheme has been generalized and extended to nonorthogonal Cartesian grids. Calculations of a variety of flow fields have demonstrated the ability of the scheme to accurately resolve developing boundary layers on coarse grids. In addition to its high accuracy, the main advantages of the present scheme are its conceptual simplicity, rigorous enforcement of both the integral and differential forms of the governing equations, and its ability to be extended to three dimensions in a straightforward manner. Work in progress is directed toward extending the scheme to three dimensions and developing a time-iterative solution technique for solving the discrete system of nonlinear equations. Finally, a scheme which uses cubic expansions to respresent the discrete primitive variables is also under development. 


\section{References}

[1] Scott, J.R. and Chang, S.C., "A New Flux Conserving Newton's Method Scheme for the TwoDimensional, Steady Navier-Stokes Equations," NASA TM 106160, June, 1993 (Accepted for publication in the International Journal of Computational Fluid Dynamics).

[2] Chang, S.C., "The Method of Space-Time Conservation Element and Solution Element - A New Approach for Solving the Navier-Stokes and Euler Equations," Submitted to the Journal of Computational Physics. (First published as "New Developments in the Method of Space-Time Conservation Element and Solution Element - Applications to the Euler and Navier-Stokes Equations," NASA TM 106226, August, 1993.)

[3] Scott, J.R., “A New Flux-Conserving Numerical Scheme for the Steady, Incompressible NavierStokes Equations," NASA TM 106520, April, 1994 (Submitted to the International Journal of Computational Fluid Dynamics).

[4] Chang, S.C. and To, W.M., "A New Numerical Framework for Solving Conservation Laws - The Method of Space-Time Conservation Element and Solution Element," NASA TM 104495, August, 1991.

[5] Anderson, D.A., Tannehill, J.C., and Pletcher, R.H., Computational Fluid Mechanics and Beat Transfer, (Hemisphere, 1984), pp. 190-193.

[6] Srinivasan, K. and Rubin, S.G., "Segmented Multigrid Domain Decomposition Procedure For Incompressible Viscous Flows", International Journal For Numerical Methods in Fluids, Vol. 15, pp. 1333-1355, 1992.

[7] Schlichting, H., Boundary-Layer Theory, 7th. ed., McGraw-Hill Series in Mechanical Engineering, Holman, J.P., Consulting Ed., (McGraw-Hill, 1979), pp. 135-140.

[8] Dill, L.H. and Himansu, A., "Development and Validation of Algorithms for the Incompressible Navier-Stokes Equations Based upon the Space Time Solution (STS) Element Method", NASA Contractor Report NAS3-27014.

[9] Messiter, A.F., "Boundary-Layer Flow Near The Trailing Edge of a Flat Plate", SIAM J. Appl. Math., 18, 1, 241 - 257.
[10] Stewartson, K., "On the flow near the trailing eige of a flat plate", II, Mathematika, 16, 106 $-121$.

[11] Scott, J.R., Dill, L.H., Oyediran, A., and Greenspan, B., "A New High-Order Numerical Method for Solving Ordinary Differential Equations", NASA TM in preparation.

[12] Chang, S.C. and To, W.M., "A Brief Description of a New Numerical Framework for Solving Conservation Laws - The Method of Space-Time Conservation Element and Solution Element," Froceedings of the Thirteenth International Conference on Numerical Methods in Fluid Dynamics, Rome, Italy, 1992, Napolitano, M. and Sabetta, F., eds., Lecture Notes in Physics 414, SpringerVerlag. Also published as NASA TM 105757.

\section{Appendix}

\section{Local Constraints:}

Conservation of Mass

$$
\begin{aligned}
u_{1,0}+v_{0,1} & =0 \\
2 u_{2,0}+v_{1,1} & =0 \\
2 v_{0,2}+u_{1,2} & =0
\end{aligned}
$$

Conservation of $x$-Momentum

$$
\begin{gathered}
u_{1,0} u_{0,0}+u_{0,1} v_{0,0}+p_{1,0} \\
-\frac{1}{R e}\left[\left(2 u_{0,0}+v_{1,1}\right)+\frac{2}{3}\left(4 u_{2,0}-v_{1,1}\right)\right]=0 \\
2\left(2 u_{0,0} u_{2,0}+u_{1,0}^{2}+p_{2,0}\right)+u_{1,1} v_{0,0} \\
+v_{1,1} u_{0,0}+u_{1,0} v_{0,1}+u_{0,1} v_{1,0}=0 \\
2\left(u_{0,0} v_{0,2}+v_{0,0} u_{0,2}+v_{0,1} u_{0,1}\right) \\
+2 u_{1,1} u_{0,0}+2 u_{1,0} u_{0,1}+p_{1,1}=0
\end{gathered}
$$

Conservation of $y$-Momentum

$$
\begin{gathered}
u_{0,0} v_{2,0}+v_{0,0} v_{0,1}+p_{0,1} \\
-\frac{1}{R e}\left[\left(2 v_{2,0}+u_{1,1}\right)+\frac{2}{3}\left(4 v_{0,2}-u_{1,2}\right)\right]=0
\end{gathered}
$$




$$
\begin{gathered}
2\left(u_{0,0} v_{2,0}+v_{0,0} u_{2,0}+v_{1,0} u_{1,0}\right) \\
+2 v_{1,1} v_{0,0}+2 v_{1,0} v_{0,1}+p_{1,1}=0 \\
2\left(2 v_{0,0} v_{0,2}+v_{0,1}^{2}+p_{0,2}\right)+u_{1,2} v_{0,0} \\
+v_{1,1} u_{0,0}+u_{1,0} v_{0,1}+u_{0,1} v_{1,0}=0
\end{gathered}
$$

General Eliminated Variables:

$$
\begin{gathered}
v_{0,1}=-u_{1,0} \\
v_{1,1}=-2 u_{2,0} \\
u_{1,1}=-2 v_{0,2} \\
p_{1,0}= \\
-u_{1,0} u_{0,0}-u_{0,1} v_{0,0}+\frac{2}{R e}\left(u_{2,0}+u_{0,2}\right) \\
p_{0,1}= \\
-v_{1,0} u_{0,0}+u_{1,0} v_{0,0}+\frac{2}{R e}\left(v_{2,0}+v_{0,2}\right) \\
p_{0,2}= \\
u_{0,0} u_{2,0}-v_{0,0} v_{0,0}-\frac{1}{2}\left(u_{1,0}^{2}+u_{0,1} v_{1,0}\right)
\end{gathered}
$$

\section{Constant-Pressure-Gradient Scheme:}

Local Constraints:

$$
\begin{array}{r}
p_{2,0}=v_{0,0} v_{0,2}-u_{0,0} u_{2,0} \\
-\frac{1}{2}\left(u_{1,0}^{2}+u_{0,1} v_{1,0}\right)=0 \\
p_{1,1}=2\left(u_{0,0} v_{0,2}-v_{0,0} u_{0,2}\right)=0 \\
p_{1,1}=2\left(v_{0,0} u_{2,0}-u_{0,0} v_{2,0}\right)=0
\end{array}
$$

\section{General Scheme:}

Local Constraint:

$$
\begin{gathered}
\left(u_{0,0} v_{0,2}-v_{0,0} u_{0,2}\right) \\
-\left(v_{0,0} u_{3,0}-u_{0,0} v_{2,0}\right)=0
\end{gathered}
$$

Additional Eliminated Variables:

$$
\begin{gathered}
p_{2,0}=v_{0,0} v_{0,2}-u_{0,0} u_{2,0} \\
-\frac{1}{2}\left(u_{1,0}^{2}+u_{0,2} v_{1,0}\right)
\end{gathered}
$$

Flux Vector Components:

\section{Conservation of Mass}

$$
\begin{gathered}
h_{0,0}^{x}=u_{0,0} \\
h_{1,0}^{x}=u_{1,0} \\
h_{0,1}^{x}=u_{0,1} \\
h_{2,0}^{x}=u_{3,0} \\
h_{1,1}^{x}=-2 h_{0,2}^{y}=-2 v_{0,2} \\
h_{0,2}^{x}=u_{0,2}
\end{gathered}
$$

$$
h_{0,0}^{y}=v_{0,0}
$$

$$
h_{0,1}^{y}=-h_{1,0}^{x}=-u_{1,0}
$$

$$
h_{1,1}^{y}=-2 h_{2,0}^{x}=-2 u_{2,0}
$$

$$
h_{0,2}^{y}=v_{0,2}
$$




$$
\begin{aligned}
& h_{0,0}^{x}=u_{0,0}^{2}+p_{0,0}-\frac{2}{R e} u_{1,0} \\
& h_{1,0}^{x}=-h_{0,1}^{y}= \\
& -v_{0,0} u_{0,1}+u_{0,0} u_{1,0}+\frac{2}{R e}\left(u_{0,2}-u_{2,0}\right) \\
& h_{0,1}^{x}=2 u_{0,0} u_{0,1}+v_{0,0} u_{1,0}-u_{0,0} v_{1,0} \\
& +\frac{2}{R e}\left(v_{2,0}+3 v_{0,2}\right) \\
& h_{2,0}^{x}=-\frac{1}{2} h_{1,1}^{y}= \\
& u_{0,0} u_{2,0}+v_{0,0} v_{0,2}+\frac{1}{2} u_{1,0}^{2}-\frac{1}{2} u_{0,1} v_{1,0} \\
& h_{1,1}^{x}=-2 h_{0,2}^{y}= \\
& -2\left(u_{0,0} v_{0,2}+v_{0,0} u_{0,2}-u_{1,0} u_{0,2}\right) \\
& h_{0,2}^{x}=2 u_{0,0} u_{0,2}+u_{0,0} u_{2,0}-v_{0,0} v_{0,2} \\
& +u_{0,1}^{2}-\frac{1}{2} u_{1,0}^{2}-\frac{1}{2} u_{0,1} v_{1,0} \\
& h_{0,0}^{y}=u_{0,0} v_{0,0}-\frac{1}{R e}\left(u_{0,1}+v_{1,0}\right) \\
& h_{1,0}^{y}=u_{0,0} v_{1,0}+v_{0,0} u_{1,0}-\frac{2}{R e}\left(v_{2,0}-v_{0,2}\right) \\
& h_{0,1}^{y}=v_{0,0} u_{0,1}-u_{0,0} u_{1,0}-\frac{2}{R e}\left(u_{0,2}-u_{2,0}\right) \\
& h_{2,0}^{y}=u_{0,0} v_{2,0}+v_{0,0} u_{2,0}+v_{1,0} u_{1,0} \\
& h_{1,1}^{y}=-2 u_{0,0} u_{2,0}-2 v_{0,0} v_{0,2} \\
& -u_{1,0}^{2}+u_{0,1} v_{1,0}
\end{aligned}
$$




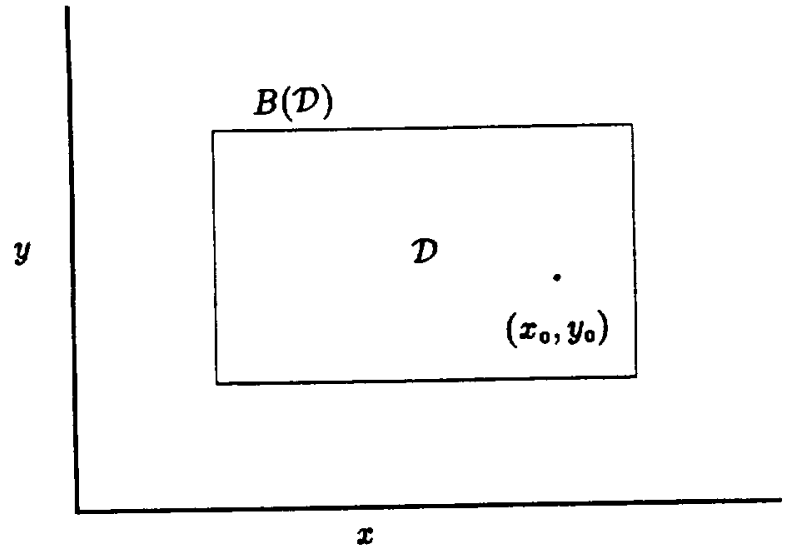

Figure 1. Rectangular domain for boundary value problem (2.2).

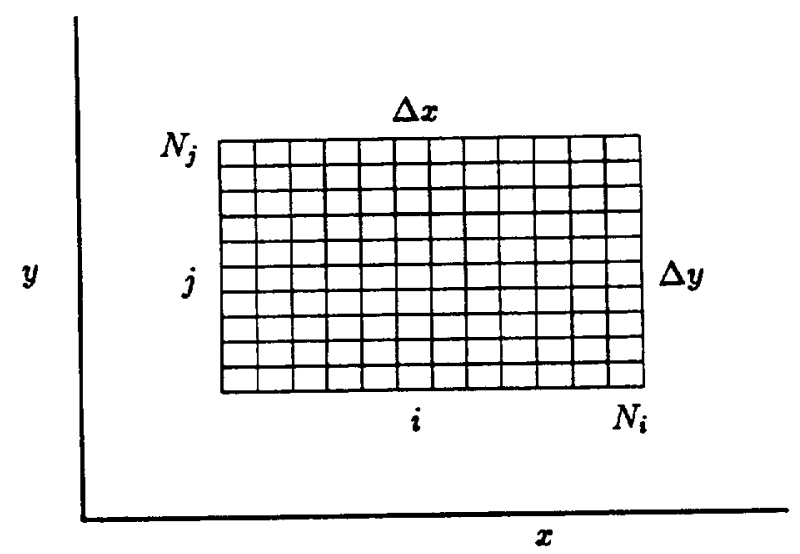

Figure 2. Discretization of domain $\mathcal{D}$.

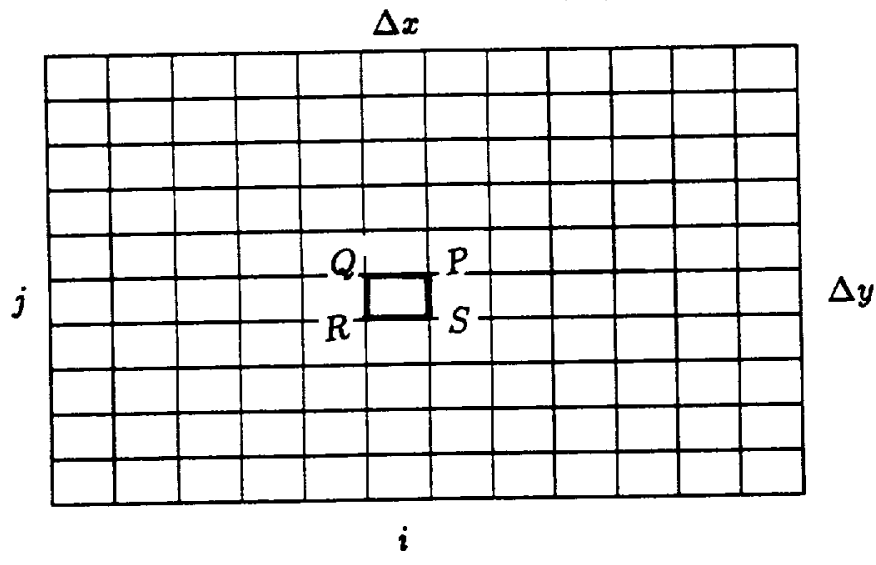

Figure 3. Cell orientation for flux expressions.

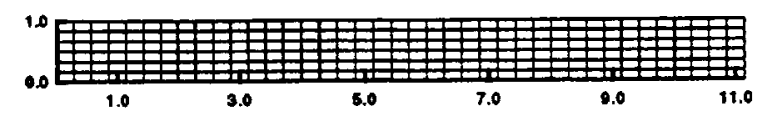

Figure 4. Channel geometry and discretization.

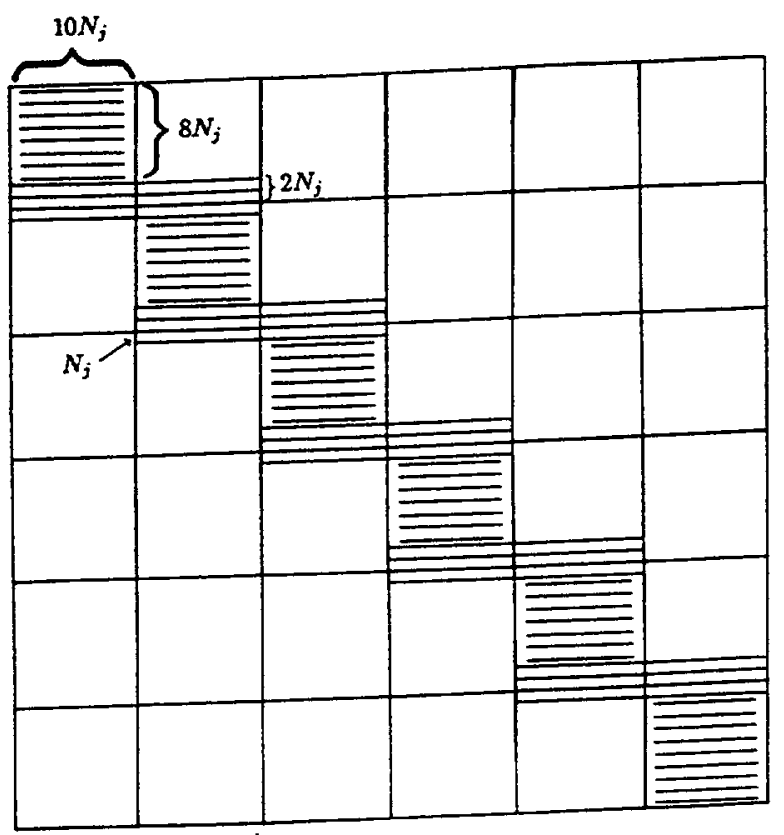

Figure 5. Jacobian matrix structure.

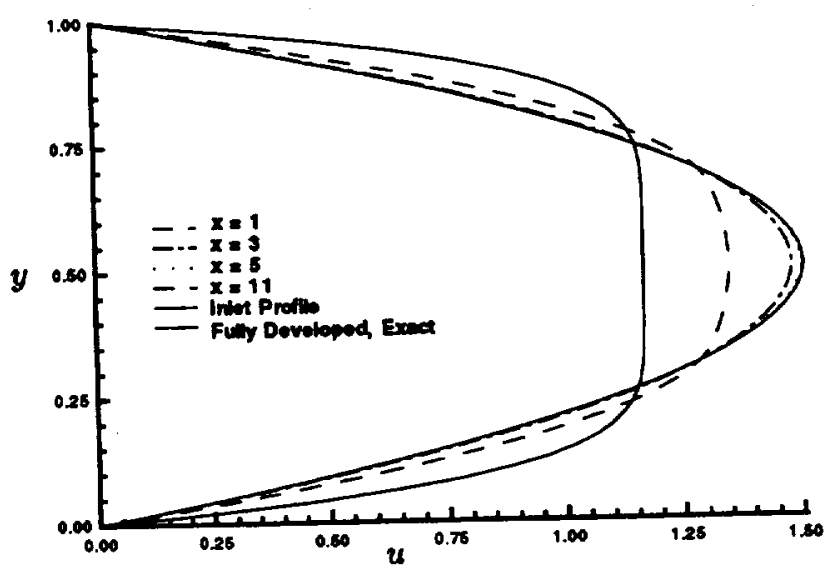

Figure 6. Developing channel flow at $1,3,5$, and 11 channel heights downstream. $R e=100.39 \times 6$ grid. 


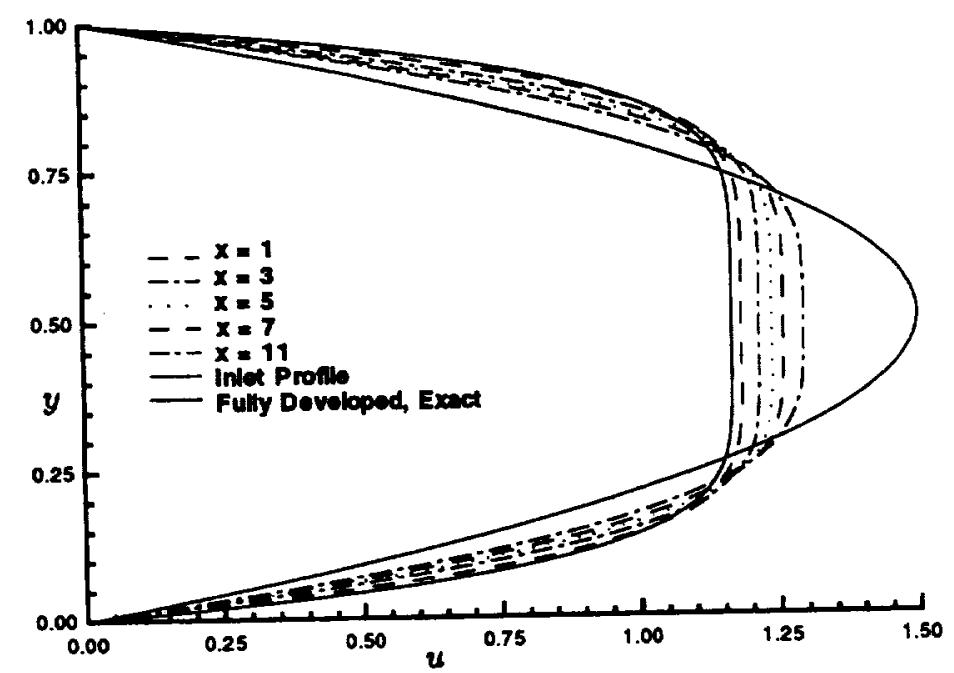

Figure 7. Developing channel flow at $1,3,5,7$, and 11 channel heights downstream. $R e=2000.39 \times 10$ grid.

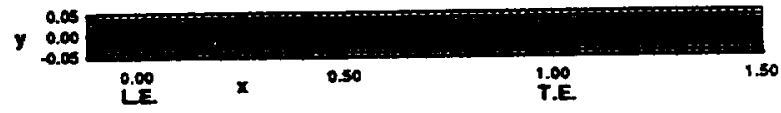

Figure 8. Computational grid for a flat plate airfoil.

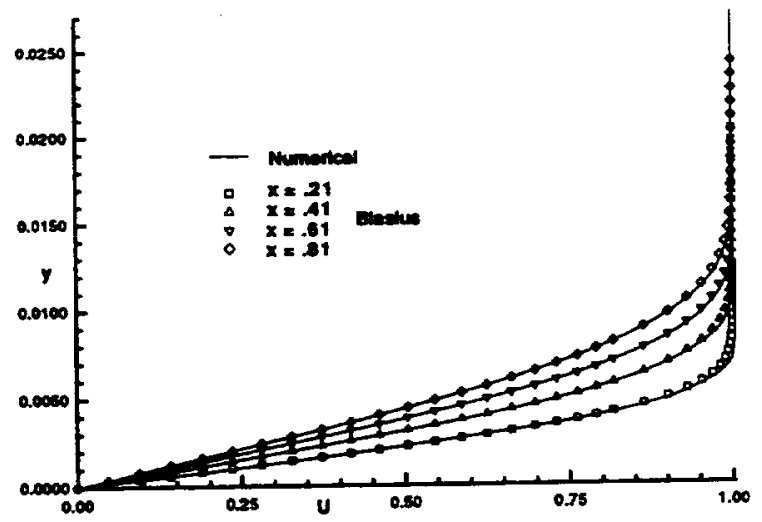

Figure 9. Developing boundary layer on a flat plate airfoil. $R e=100,000.81 \times 20$ grid. $12.5 \%$ exponential $y$ stretching.

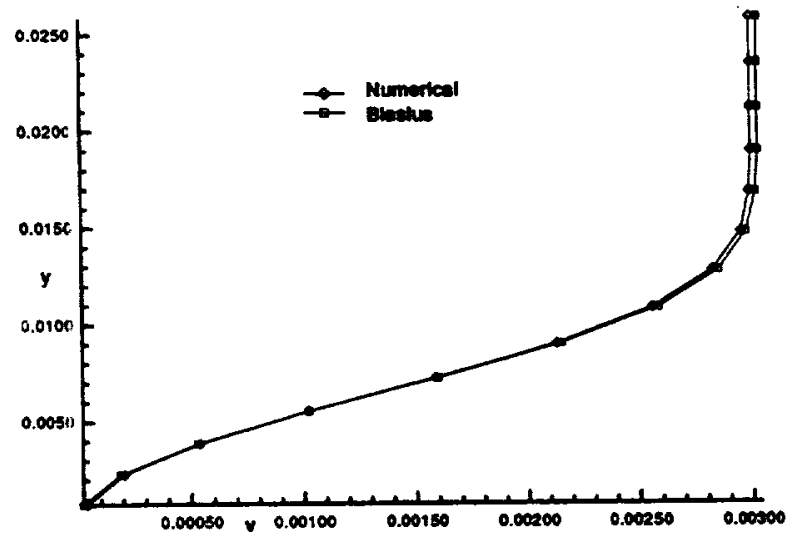

Figure 10. Comparison of $v$ velocity component with Blasius solution. $R e=100,000.110 \times 28$ grid.

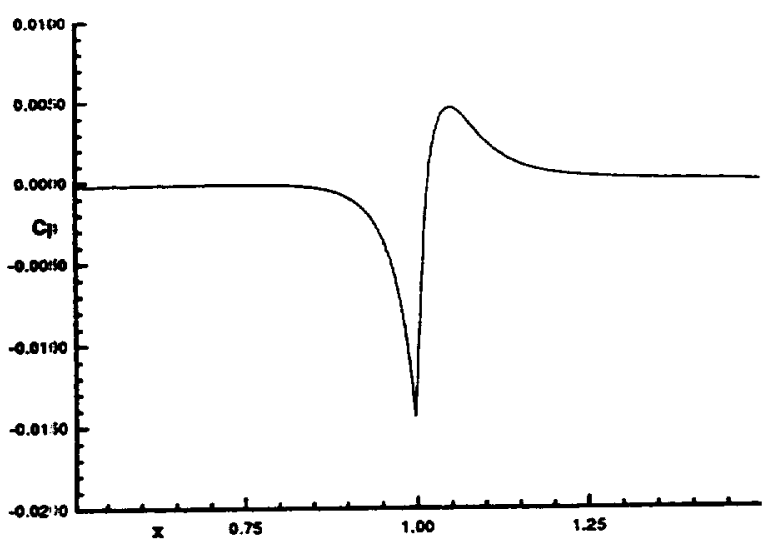

Figure: 11. Pressure coefficient in the trailing edge region. $R e=100,000.110 \times 22$ grid.

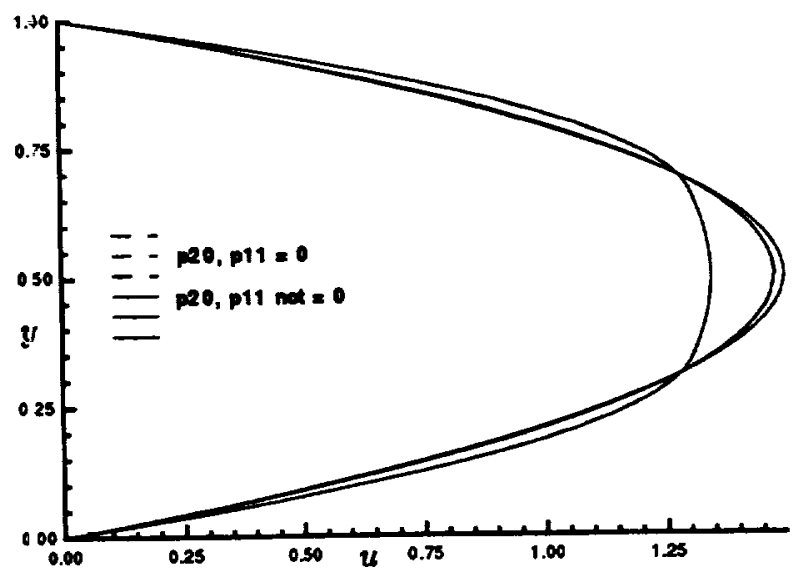

Figure 12. Comparison of numerical results from the gener:alized scheme and the constant-pressure-gradient scherre. Channel flow at $R e=100 . x=1,3,5$. 


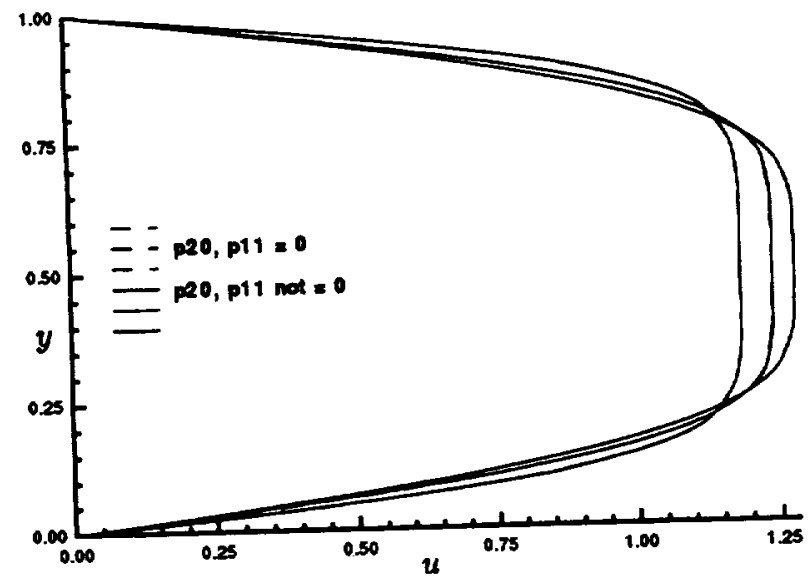

Figure 13. Comparison of numerical results from the generalized scheme and the constant-pressure-gradient scheme. Channel flow at $R e=2000 . x=1,5,9$.

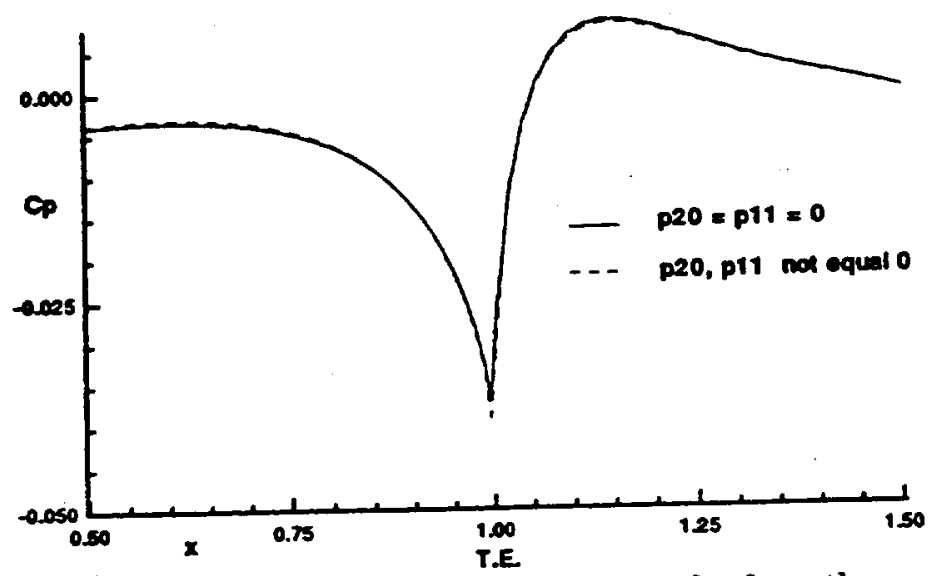

Figure 14. Comparison of numerical results from the generalized scheme and the constant-pressure-gradient scheme. Pressure coefficient in the trailing edge region of a flat plate airfoil. $R e=7.500$.

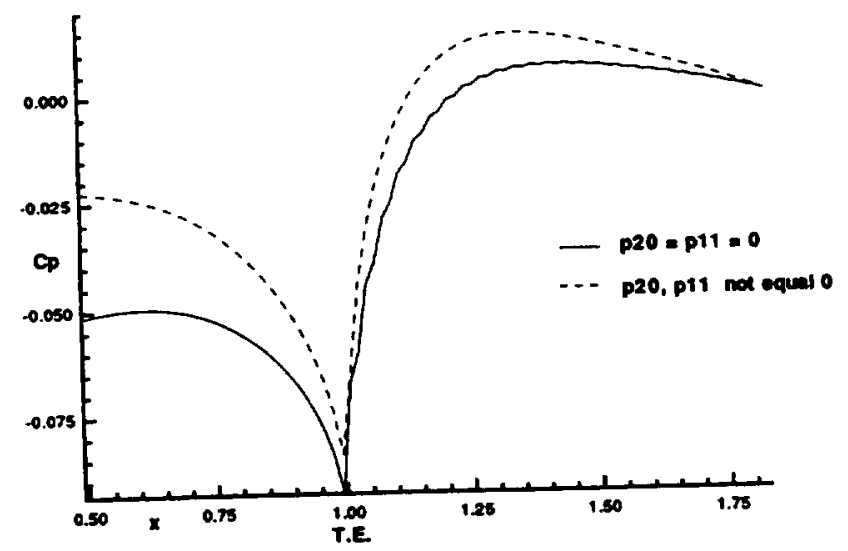

Figure 15. Comparison of numerical results from the generalized scheme and the constant-pressure-gradient scheme. Pressure coefficient in the trailing edge region of a flat plate airfoil. $R e=1000$.

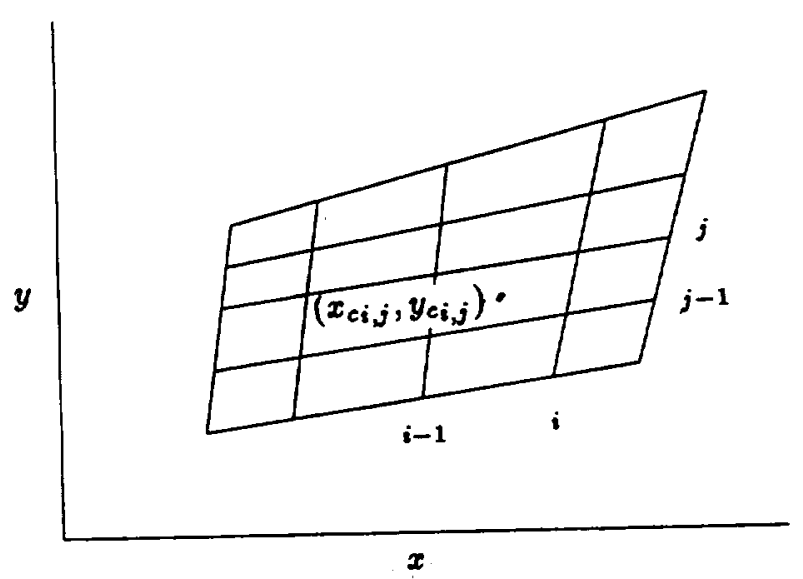

Figure 16. Nonorthogonal Cartesian grid.

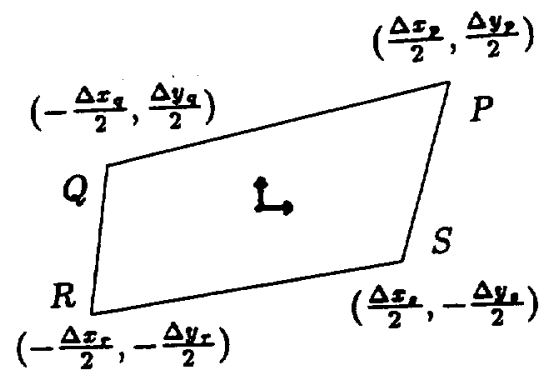

Figure 17. Local coordinate system for flux evaluation.

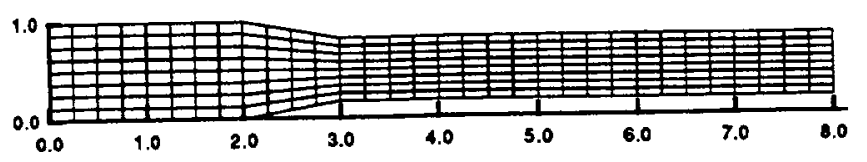

Figure 18 a. Computational grid for a converging channel. Ramp angle $=10$ degrees. $32 \times 8$ grid. 


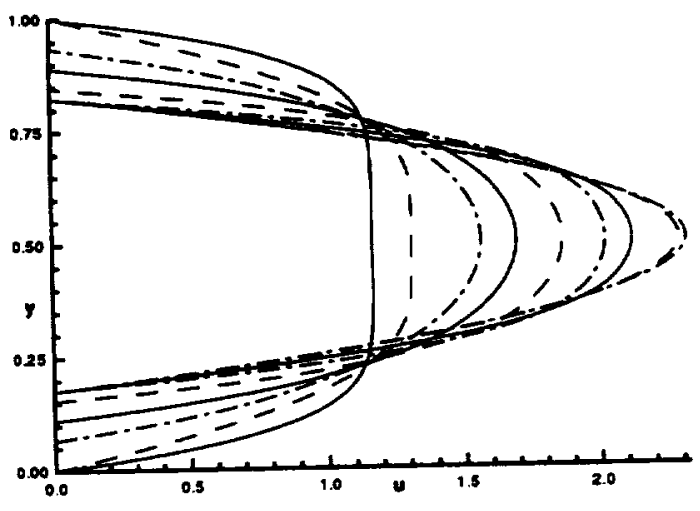

Figure $18 \mathrm{~b}$. Developing flow in a channel with a converging section. $R e=100$.

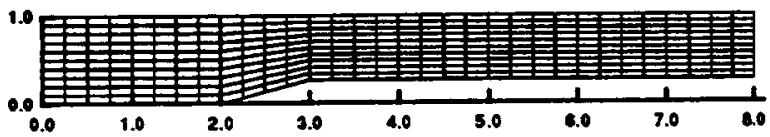

Figure 19 a. Computational grid for a channel with a 15 degree ramp. $32 \times 10$ grid.

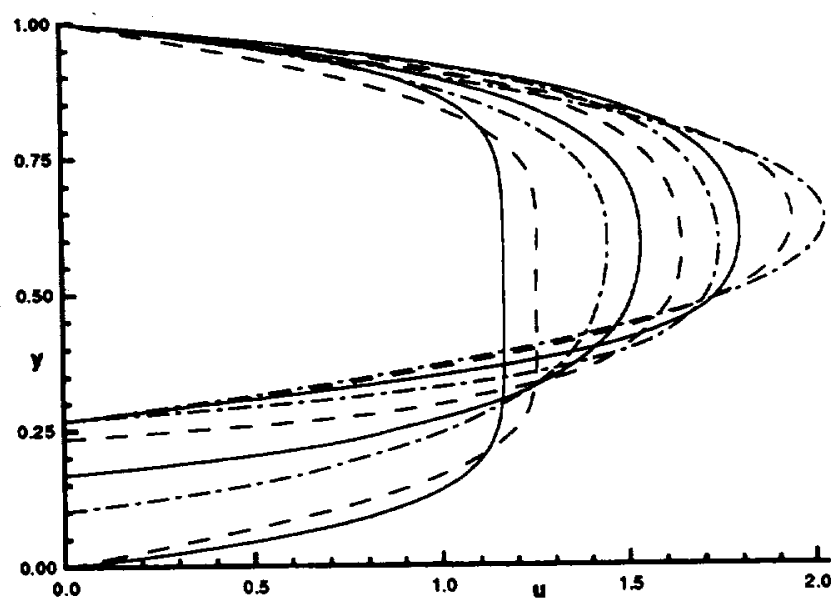

Figure $19 \mathrm{~b}$. Developing flow in a channel with a 15 degree ramp. $R e=200$.

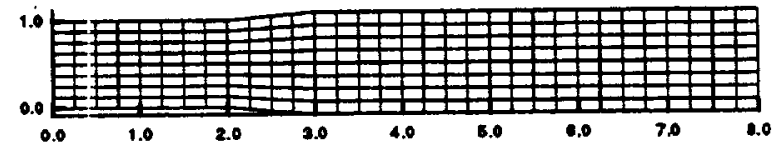

Figure 20 a. Computational grid for a diverging channel. Pramp angle $=5$ degrees. $32 \times 8$ grid.

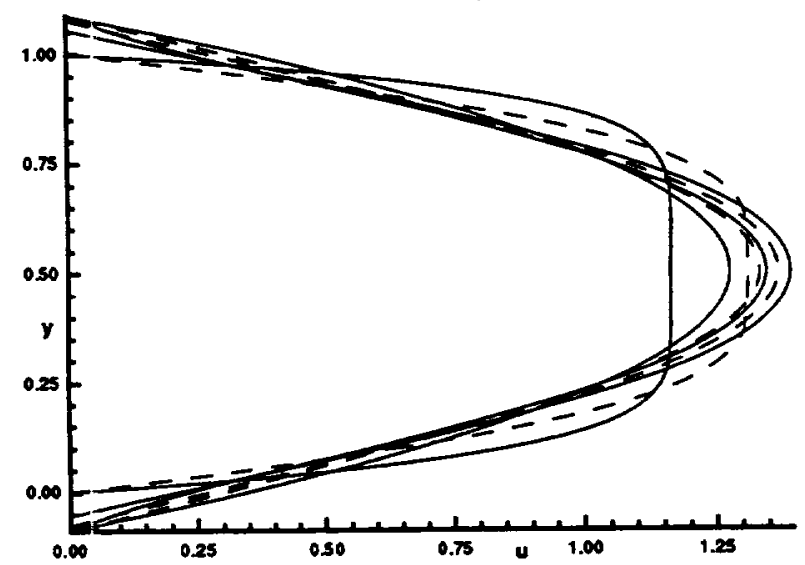

Figure $20 \mathrm{~b}$. Developing flow in a diverging channel with a 5 degree ramp. $R e=100$.

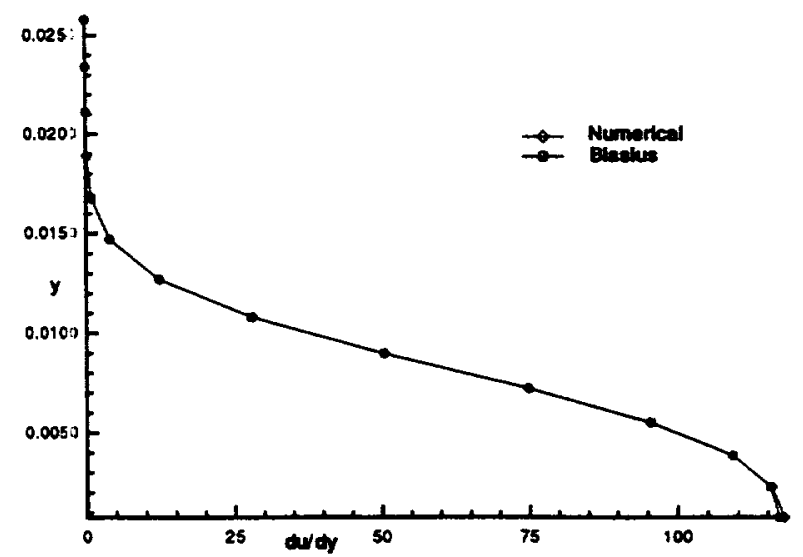

Figure 21. Comparison between $u_{0,1}$ and the first $y$ derivative of the Blasius solution. $x=.805$. $110 \times 28$ grid. $R e=100,000$. 


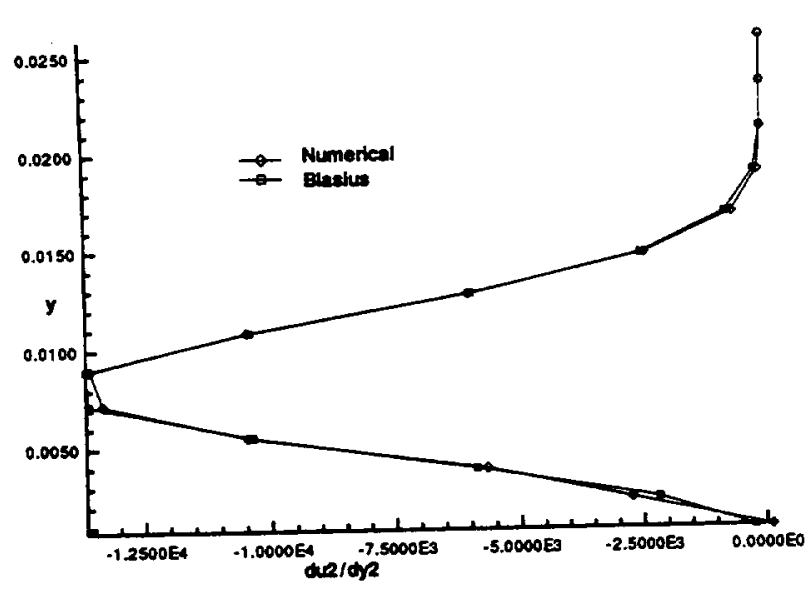

Figure 22. Comparison between $2 u_{0,2}$ and the second $y$ derivative of the Blasius solution. $x=.805 .110 x$ 28 grid. $R e=100,000$.
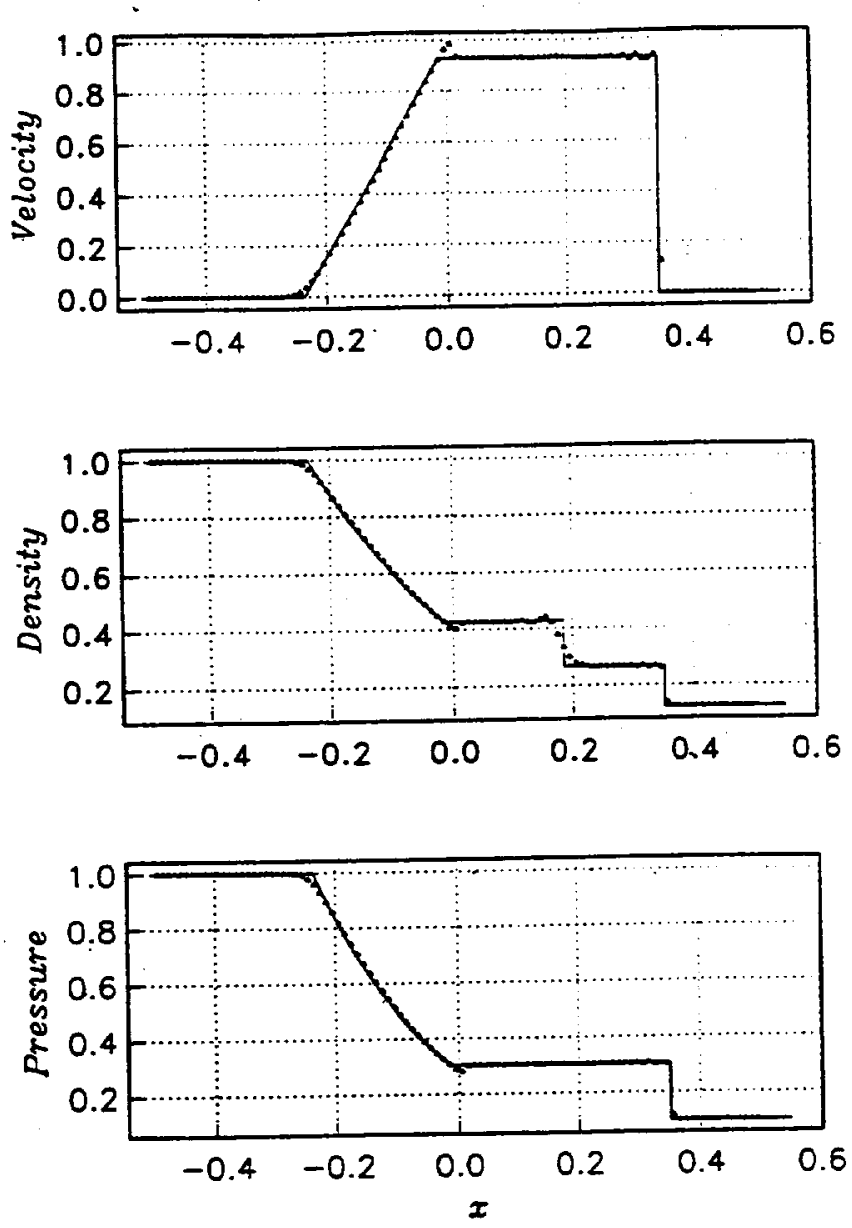

Figure 23. Navier-Stokes solution to the shock tube problem with viscosity. $R e=12,000$. 


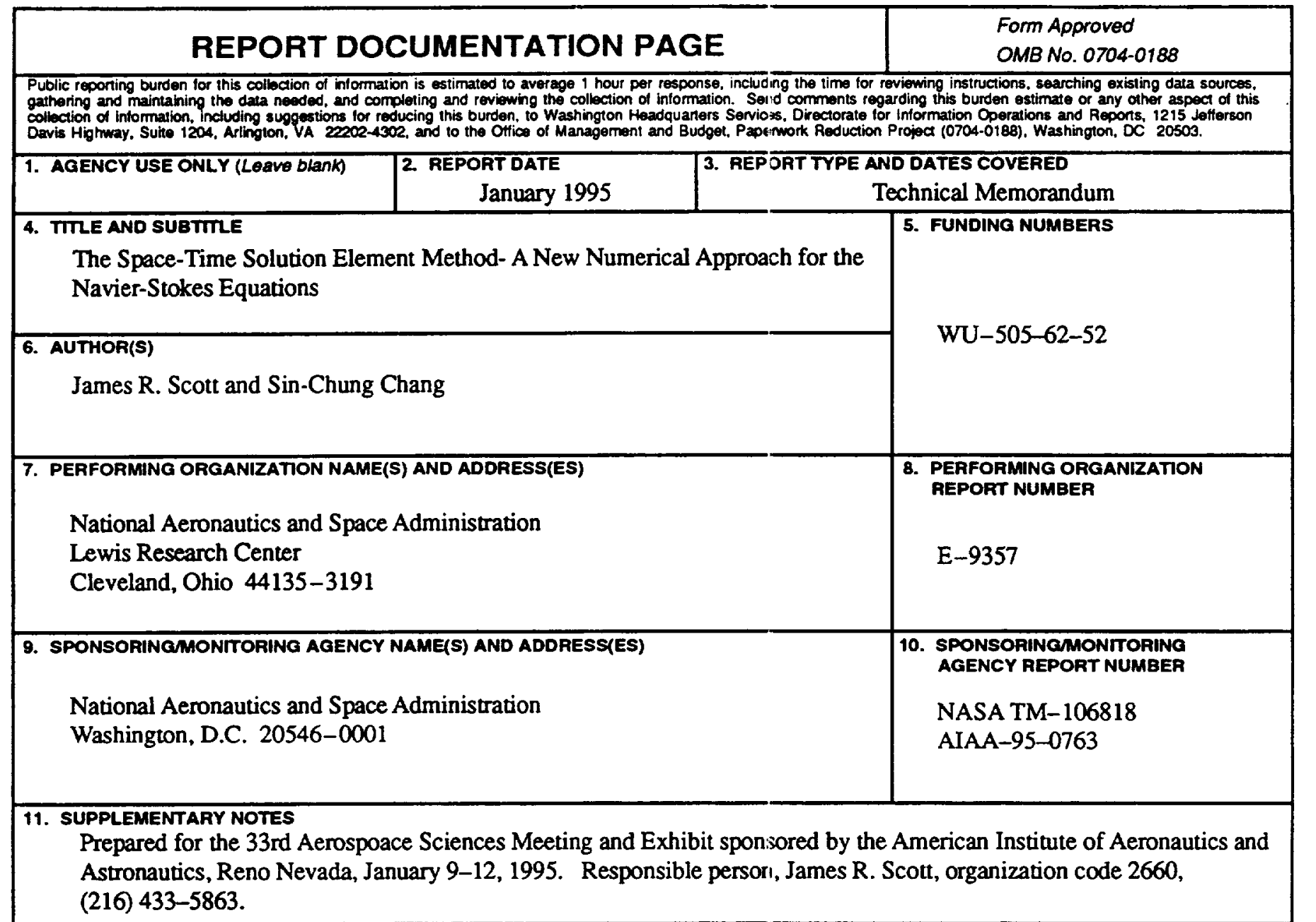

\begin{tabular}{l|l} 
12a. DISTRIBUTIONAAVALABILTYY STATEMENT & 12b. DISTRIBUTION CODE
\end{tabular}

Unclassified - Unlimited

Subject Categories 02, 34, 64

This publication is available from the NASA Center for Aerospace Information, (301) 621-0390.

13. ABSTRACT (Maximum 200 words)

This paper is one of a series of papers describing the development of a new numerical method for the Navier-Stokes equations. Unlike conventional numerical methods, the current methoi concentrates on the discrete simulation of both the integral and differential forms of the Navier-Stokes equations. Conser ration of mass, momentum, and energy in spacetime is explicitly provided for through a rigorous enforcement of both he integral and differential forms of the governing conservation laws. Using local polynomial expansions to represent the discrete primitive variables on each cell, fluxes at cell interfaces are evaluated and balanced using exact functional expressions. No interpolation or flux limiters are required. Because of the generality of the current method, it applies equally to the steady and unsteady Navier-Stokes equations. In this paper,we generalize and extend the authors' 2-D, ste.ady state implicit scheme. A general closure methodology is presented so that all terms up through a given order in the local expansions may be retained. The scheme is also extended to nonorthogonal Cartesian grids. Numerous flow fields are computed and results are compared with known solutions, The high accuracy of the scheme is demonstrated through its ability to accurately resolve developing boundary layers on coarse grids. Finally, we discuss applications of th: current method to the unsteady Navier-Stokes equations.

\begin{tabular}{|c|c|}
\hline $\begin{array}{l}\text { 14. SUBJECT TERMS } \\
\text { Navier-Stokes; Conserva } \\
\text { Flux; Flux-Conserving; }\end{array}$ & Law; \\
\hline $\begin{array}{l}\text { 17. SECURTY CLASSIFICATION } \\
\text { OF REPORT } \\
\text { Unclassified }\end{array}$ & $\begin{array}{l}\text { 18. SECURTY CLASSIFICATION } \\
\text { OF THIS PACE } \\
\text { Unclassified }\end{array}$ \\
\hline
\end{tabular}

\begin{tabular}{|c|c|}
\hline \multirow[t]{2}{*}{ lement; Conservation Element: } & $\begin{array}{c}\text { 15. NUMBER OF PAGES } \\
19\end{array}$ \\
\hline & $\begin{array}{r}\text { 16. PRICE CODE } \\
\mathrm{A03}\end{array}$ \\
\hline $\begin{array}{l}\text { 19. SECURITY CLASSIFICATION } \\
\text { OF ABSTRACT } \\
\text { U"nclassified }\end{array}$ & 20. LIMITATION OF ABSTRACT \\
\hline
\end{tabular}

